Interfaces and Free Boundaries 16 (2014), 65-104

DOI 10.4171/IFB/314

\title{
A Hilbert expansion method for the rigorous sharp interface limit of the generalized Cahn-Hilliard equation
}

\author{
Dimitra C. Antonopoulou \\ Department of Mathematics and Applied Mathematics, University of Crete, GR-71409 Heraklion, \\ Greece, and \\ Institute of Applied and Computational Mathematics, FORTH, GR-711 10 Heraklion, Greece \\ E-mail:danton@tem.uoc.gr \\ GEORGIA KARALI \\ Department of Mathematics and Applied Mathematics, University of Crete, GR-71409 Heraklion, \\ Greece, and \\ Institute of Applied and Computational Mathematics, FORTH, GR-711 10 Heraklion, Greece \\ E-mail: gkarali@tem.uoc.gr \\ ENZA ORLANDI \\ Dipartimento di Matematica e Fisica, Università di Roma Tre, L.go S. Murialdo 1, 00146 Roma, \\ Italy \\ E-mail: orlandi@mat.uniroma3.it
}

[Received 4 January 2013 and in revised form 28 November 2013]

\begin{abstract}
We consider Cahn-Hilliard equations with external forcing terms. Energy decreasing and mass conservation might not hold. We show that level surfaces of the solutions of such generalized Cahn-Hilliard equations tend to the solutions of a moving boundary problem under the assumption that classical solution of the latter exists. Our strategy is to construct approximate solutions of the generalized Cahn-Hilliard equation by the Hilbert expansion method used in kinetic theory and proposed for the standard Cahn-Hilliard equation, by Carlen, Carvalho and Orlandi, [14]. The constructed approximate solutions allow to derive rigorously the sharp interface limit of the generalized Cahn-Hilliard equations and higher order corrections to the limiting motion. We then estimate the difference between the true solutions and the approximate solutions by spectral analysis, as in [1].
\end{abstract}

2010 Mathematics Subject Classification: Primary 35K55, 35K77.

Keywords: Cahn-Hilliard equation, forcing, sharp interface limit, Hilbert expansion.

\section{Introduction}

In this paper, we apply an alternative method to matched asymptotic expansions, developed by Carlen, Carvalho and Orlandi, in [14], which allows the study of the sharp interface limit for the generalized Cahn-Hilliard equation, and derive higher order corrections to this limit. The method is based on the Hilbert expansion used in kinetic theory; we refer to [14] where the analogy is explained. We start by recalling some back ground regarding the Cahn-Hilliard equation and the results obtained in [14]. 


\subsection{The Cahn-Hilliard equation}

Let $\Omega$ be a bounded domain in $\mathbb{R}^{2}$. The restriction of the analysis to two dimensions is made only for simplicity. Let $m=m(x, t)$ be an integrable function on $\Omega$ which represents the value of a conserved "order parameter" at $x$ in $\Omega$ at time $t$. The order parameter is conserved in the sense that $\int_{\Omega} m(x, t) \mathrm{d} x$ is independent of $t$. Therefore, the evolution equation for $m$ can be written in the form

$$
\partial_{t} m(x, t)=\nabla \cdot \vec{J}(x, t),
$$

where the current $\vec{J}$ is orthogonal to the outer normal of the boundary of $\Omega$. We take

$$
\vec{J}(x, t)=\sigma(m(x, t)) \nabla \mu(x, t),
$$

where $\sigma(m)$ is the mobility and $\mu(x, t)$ is the chemical potential of $x$ at time $t$. The mobility is positive and the chemical potential is defined as the $L^{2}(\Omega)$ Frechet derivative of a free energy functional F:

$$
\mu(x)=\frac{\delta \mathcal{F}}{\delta m}(x) .
$$

The simplest and most familiar example is the so called Cahn-Hilliard equation. It results by setting $\sigma(m):=1$, i.e., constant mobility, and

$$
\mathcal{F}(m):=\frac{1}{2} \int_{\Omega}|\nabla m(x)|^{2} \mathrm{~d} x+\frac{1}{4} \int_{\Omega}\left(m^{2}(x)-1\right)^{2} \mathrm{~d} x .
$$

This leads to the evolution equation

$$
\partial_{t} m(x, t)=\Delta(-\Delta m(x, t)+f(m(x, t))),
$$

where

$$
f(m)=m^{3}-m .
$$

Different choices of $f$ can be made, provided they are derivatives of a double well, smooth enough potential, with equal absolute minima. If $m(x, t)$ is a solution of this equation, then

$$
\frac{\mathrm{d}}{\mathrm{d} t} \mathcal{F}(m(\cdot, t))=-\int_{\Omega}|\vec{J}(x, t)|^{2} \mathrm{~d} x,
$$

and thus, evolution decreases the free energy. The minimizers of the free energy are the constant functions $m= \pm 1$. These minimizers represent the "pure phases" of the system. However, unless the initial condition $m_{0}$ happens to satisfy $\int_{\Omega} m_{0}(x) \mathrm{d} x= \pm|\Omega|$, these "pure phases" cannot be reached due to the mass conservation law. Instead, what will eventually be produced is a region in which $m \approx+1$ while $m \approx-1$ in its complement, with smooth transition across its boundary. This phenomenon is referred to as phase segregation, where the aforementioned boundary is the interface between the two phases. If we "stand far enough back" from $\Omega$, all we can observe is the interface's shape since the structure across the interface is placed on an invisibly small scale.

The evolution of $m$ under the Cahn Hilliard equation, or another equation of this type, drives a very slow evolution of the interface. More specifically, let $\varepsilon$ be a small parameter, and introduce the new variables $\tau$ and $\xi$ by

$$
\tau:=\varepsilon^{3} t \quad \text { and } \quad \xi:=\varepsilon x .
$$


Then of course it follows

$$
\partial_{t}=\varepsilon^{3} \partial_{\tau} \quad \text { and } \quad \partial_{x}=\varepsilon \partial_{\xi} .
$$

Hence, if $m(x, t)$ is a solution of the Cahn-Hilliard equation and we define $m^{\varepsilon}(\xi, \tau):=$ $m(x(\xi), t(\tau))$ then we obtain

$$
\partial_{\tau} m^{\varepsilon}(\xi, \tau)=\Delta_{\xi}\left(-\varepsilon \Delta_{\xi} m^{\varepsilon}(\xi, \tau)+\frac{1}{\varepsilon} f\left(m^{\varepsilon}(\xi, \tau)\right)\right) .
$$

If we think of $\varepsilon$ as representing the inverse of a large length scale, the variable $\xi$ will be dimensionless. The dimensionless variables are "slow" and the original variables "fast" for small $\varepsilon$. In what follows, we keep the notation $\xi$ for the slow spatial variables, but we drop the use of $\tau$ and replace it by $t$ for convenience. One should just bear in mind that now we are looking at the evolution over a very long time scale when $\varepsilon$ is small. For the reasons indicated above, it is customary to consider initial data $m_{0}(\xi)$ that is -1 in the region bounded by a smooth closed curve $\Gamma_{0}$ in $\Omega$, and +1 outside this region. At later times $t$ there will still be a fairly sharp interface between a region where $m(\xi, t) \approx+1$ and a region where $m(\xi, t) \approx-1$, centered on a smooth curve $\Gamma_{t}$. One might hope that for small values of $\varepsilon$, all information about the evolution of $m^{\varepsilon}(\xi, t)$ is contained in the evolution of the interface $\Gamma_{t}$. This is indeed the case as shown in [14]. To explain the method used in [14], let $m$ denote the set of all smooth simple closed curves in $\Omega$. As we will explain in Section 2, $m$ can be viewed as a differentiable manifold. A vector field $V$ on $m$ is a functional associating to each $\Gamma$ in $m$ a function in $C^{\infty}(\Gamma)$. This function gives the normal velocity of a point on $\Gamma$, and thus describes a "flow" on $m$. We may formally write

$$
\frac{\mathrm{d}}{\mathrm{d} t} \Gamma_{t}=V\left(\Gamma_{t}\right) .
$$

Now, given a flow on $m$, we can produce from it an evolution in $C^{\infty}(\Omega)$ through the following device: Let $m$ be any function from $m$ to $C^{\infty}(\Omega)$. We write $m(\xi, \Gamma)$ to denote $m(\Gamma)$ evaluated at $\xi \in \Omega$. We can then define a time dependent function $m(\xi, t)$ on $\Omega$ by

$$
m(\xi, t):=m\left(\xi, \Gamma_{t}\right)
$$

Notice that time dependence in $m(\xi, t)$ enters only through the evolution of $\Gamma_{t}$. Now if, for small $\varepsilon$ and sharp interface initial data, all of the information about the evolution of solutions of the CahnHilliard equation were contained in the motion of the interface, then one might hope to find a vector field $V$ on $m$ governing the evolution of the interface, and a function $m$ from $m$ to $C^{\infty}(\Omega)$ so that (1.4) defines the corresponding solution of the Cahn-Hilliard equation.

In [14], a result of this type has been proved. More specifically, a sequence of vector fields $V_{0}, V_{1}, V_{2}, \cdots$ defined on $m$ was constructed such that the interface for the solution of (1.2) satisfies (1.3) for $V:=\sum_{j=0}^{\infty} \varepsilon^{j} V_{j}$. It turned out that the leading term $V_{0}$ is the vector field generating the Mullins Sekerka flow, as one could expect from the pioneering work of Pego [33] made rigorous by Alikakos, Bates and Chen [1]. In these papers the approximate solutions were constructed by using matched asymptotic expansions which give no information on the higher order corrections to the flow. The approach introduced in [14] unable to determine at any given order the velocity of the flow.

Let us fix a number $S>0$ that will later be interpreted as a "surface tension", denote by $K(\xi) \equiv K(\xi, \Gamma)$ the curvature at $\xi \in \Gamma$ and by $v$ the unit outward normal either to $\partial \Omega$ or to $\Gamma$. 
Further, for each $\Gamma$ in $m$, let $\mu$ be the solution of

$$
\Delta \mu(\xi)=0 \text { for } \xi \in \Omega \backslash \Gamma,
$$

subject to the boundary conditions

$$
\mu(\xi)=S\left(K(\xi)-\frac{2 \pi}{|\Gamma|}\right) \quad \text { on } \quad \Gamma, \quad \partial_{\nu} \mu=0 \quad \text { on } \quad \partial \Omega,
$$

where $|\Gamma|$ denotes the arc length of $\Gamma$ and $\partial_{\nu}$ is the outward normal derivative to $\partial \Omega$. Now define $V_{0}(\Gamma)$ as the real valued function on $\Gamma$ given by

$$
V_{0}(\xi, \Gamma):=\frac{1}{2}\left[\partial_{\nu} \mu\right]_{\Gamma}(\xi), \quad \xi \in \Gamma,
$$

where the brackets on the right-hand side denote the jump of the normal derivative across $\Gamma$. In this way one defines a vector field on $m$ which generates a flow known as the Mullins-Sekerka flow. For the local existence of a unique smooth solution of the free boundary problem (1.5), (1.6) and (1.7) see Chen, [16] and Escher and Simonett, [21]. As it is well known, the Mullins-Sekerka flow conserves the area enclosed by $\Gamma_{t}$ and decreases the arc length of $\Gamma_{t}$.

The higher order terms in $\sum_{j=0} \varepsilon^{j} V_{j}$ are more complicated. In [14], $V_{1}$ which is the next correction to $V_{0}$ was computed and a general technique of calculating all the higher order terms has been presented. The description of $V_{1}$, like that of $V_{0}$, is in the context of potential theory.

\subsection{The E-dependent generalized Cahn-Hilliard equation}

We consider the generalized Cahn-Hilliard equation of the following type

$$
\partial_{t} m^{\varepsilon}(\xi, t)=\Delta\left(-\varepsilon \Delta m^{\varepsilon}(\xi, t)+\frac{f\left(m^{\varepsilon}(\xi, t)\right)}{\varepsilon}-G_{2}(\xi ; \varepsilon)\right)+G_{1}(\xi ; \varepsilon), \quad \xi \text { in } \quad \Omega, \quad t>0,
$$

where $\Delta$ is the Neumann Laplacian operator on $\Omega$. The terms $G_{1}$ and $G_{2}$ may depend on time also. In the present analysis, we shall consider the case where $G_{1}$ and $G_{2}$ depend only on $\xi$ since our aim is to explain the main strategy in the simplest interesting setting. As it will be clear in the sequence, the proposed method is suitable for the time dependent case as well.

The term $G_{2}$ in (1.8) models general external fields, see [25, 26]. In [28] the authors apply the Kawasaki exchange dynamics to derive a modified Cahn-Hilliard equation where $G_{2}$ describes the external gravity field. When $G_{1}=0$, one can regard equation (1.8) as a particular case of the conserved phase field system with prescribed temperature, see for example [8].The free energyindependent term $G_{1}$ may describe an external mass supply, cf. [25], or [3] where $G_{1}$ was defined as a deterministic Gaussian function. Such a model is described for example in [3], in order to model spinodal decomposition in the presence of a moving particle source, as a mechanism for the formation of Liesengang bands. In addition, $G_{1}$ was introduced as a conservative white noise of thermal fluctuations cf. [26] or [17] (Cahn-Hilliard-Cook model). Existence and uniqueness of solution for the stochastic problem was established in $[6,13,18]$ while dynamics and stochastic stability were analyzed for the one-dimensional case in [5]. Furthermore, the interface stochastic motion and singular perturbation has been studied for many related models like Allen-Cahn or Ginzburg Landau and phase-field models, cf. for example [4, 23]. 
Integrating (1.8) over $\Omega$ we get

$$
\partial_{t}\left(\int_{\Omega} m^{\varepsilon}(\xi, t) d \xi\right)=\int_{\partial \Omega} \partial_{\nu}\left(-\varepsilon \Delta m^{\varepsilon}+\frac{f\left(m^{\varepsilon}\right)}{\varepsilon}-G_{2}(s ; \varepsilon)\right) d s+\int_{\Omega} G_{1}(\xi ; \varepsilon) d \xi .
$$

Therefore, $\int_{\Omega} m^{\varepsilon}(\xi, t) d \xi$ is not conserved unless the second member of (1.9) is null. Generally, due to the presence of the external force field $G_{2}$ and the external mass supply $G_{1}$, a free energy decreasing is not expected. For a mathematical analysis of the problem when $G_{2}=0$ and $G_{1}$ is in $L^{2}(\Omega)$ cf. [19, 35].

An equivalent system formulation of (1.8) is the following

$$
\begin{gathered}
\partial_{t} m^{\varepsilon}(\xi, t)=\Delta \mu^{\varepsilon}(\xi, t)+G_{1}(\xi ; \varepsilon), \\
\mu^{\varepsilon}(\xi, t)=-\varepsilon \Delta m^{\varepsilon}(\xi, t)+\frac{1}{\varepsilon} f\left(m^{\varepsilon}(\xi, t)\right)-G_{2}(\xi ; \varepsilon),
\end{gathered}
$$

where $\Delta$ is the Neumann Laplacian operator on $\Omega$. This representation will be used in our analysis. For the purposes of this paper we consider the $\varepsilon$-dependent generalized Cahn-Hilliard equation (1.8) (and equivalently the system (1.10), (1.11)) supplemented with an initial condition

$$
m^{\varepsilon}(\xi, 0)=m_{0}^{\varepsilon}(\xi) \simeq\left\{\begin{array}{ccc}
-1 & \text { on } & \Omega_{0}^{-} \\
+1 & \text { on } & \Omega_{0}^{+},
\end{array}\right.
$$

where $\Omega_{0}^{-}$is the region of $\Omega$ enclosed by a smooth closed curve $\Gamma_{0}$ and $\Omega_{0}^{+}=\Omega \backslash\left(\Omega_{0}^{-} \cup \Gamma_{0}\right)$. Thus, we are assuming that the interface is already initial formed. Further we take the following Neumann boundary conditions

$$
\partial_{\nu} m^{\varepsilon}=\partial_{\nu} \Delta m^{\varepsilon}=0 \quad \text { on } \quad \partial \Omega .
$$

We assume that the forcing terms $G_{1}$ and $G_{2}$ are sufficiently smooth, and that $\partial_{\nu} G_{2}=0$ on $\partial \Omega$ so that (1.13) becomes

$$
\partial_{\nu} m^{\varepsilon}=\partial_{\nu} \mu^{\varepsilon}=\partial_{\nu} \Delta m^{\varepsilon}=0 \quad \text { on } \partial \Omega .
$$

We dot not require

$$
\int_{\Omega} G_{1}(\xi ; \varepsilon) d \xi=0
$$

Hence, mass conservation might not hold. The precise assumptions for the forcing terms $G_{1}$ and $G_{2}$ will be given in Section 2. For sufficiently smooth initial conditions and forcing terms $G_{1}, G_{2}$, there exists a unique classical solution of the generalized Cahn-Hilliard equation. The proof is analogous to that of the homogeneous case presented in [19].

Notice that if we write (1.8) in the original not scaled variables $(x, t)$ the terms $G_{1}$ and $G_{2}$ are small perturbations of the standard Cahn-Hilliard equation. The term $G_{1}$ in the original variables $(x, t)$ is multiplied by a factor $\varepsilon^{3}$ and the term $G_{2}$ by a factor $\varepsilon$. The problem that we pose is the following. Take as in the homogeneous Cahn-Hilliard equation, initial data $m_{0}(\xi)$ like in (1.12). Due to the presence of $G_{1}$ and $G_{2}$ the constant functions $m^{\varepsilon}= \pm 1$ are not anymore stationary solutions of (1.8). But we still expect that eventually at later times $t$ there will appear a fairly sharp interface between the regions where $m^{\varepsilon}(\xi, t) \approx+1$ and where $m^{\varepsilon}(\xi, t) \approx-1$, centered on some smooth curve $\Gamma_{t}$. We prove that this is indeed the case. We derive the motion of $\Gamma_{t}$ determining the 
vector field. It turns out that the leading term $V_{0}$ in the vector field $\sum_{j=0}^{N-1} \varepsilon^{j} V_{j}\left(\Gamma_{t}^{(N)}\right)$, governing the interfacial flow (see (2.7)), is not the vector field generating the Mullins Sekerka flow appearing in the sharp limit of the homogeneous Cahn-Hilliard equation. In fact, we obtain

$$
V_{0}\left(\cdot, \Gamma_{t}\right)=V_{0}^{(0)}\left(\cdot, \Gamma_{t}\right)+\left\langle V_{0}\right\rangle_{\Gamma_{t}},
$$

where

$$
\int_{\Gamma_{t}} V_{0}^{(0)}\left(\eta, \Gamma_{t}\right) \mathrm{d} S_{\eta}=0
$$

and

$$
\left\langle V_{0}\right\rangle_{\Gamma_{t}}=\frac{1}{2\left|\Gamma_{t}\right|} \int_{\Omega} G_{1,0}(\eta) \mathrm{d} \eta, \quad t \in[0, T] .
$$

Here, and in the following, we denote by $\mathrm{d} S_{\eta}$ the element of the arc length along $\Gamma$ or $\partial \Omega$. We will indeed prove that, as $\varepsilon \rightarrow 0$ the singular limit of (1.10) and (1.11) leads to the following moving boundary problem: Given a closed curve $\Gamma^{0}$ in $\Omega$ that it is the boundary of an open set $\Omega_{0}^{-} \subset \Omega$ find a family $\left\{\Gamma_{t} \in \mathcal{M}: t \in[0, T]\right\}$ and functions $\mu(\xi, t)=\mu\left(\xi, \Gamma_{t}\right)$ for $t \in[0, T]$ and $\xi \in \Omega$ so that

$$
\begin{aligned}
& \Delta \mu(\xi, t)=-G_{1,0}(\xi) \quad \xi \in \Omega \backslash \Gamma_{t}, \quad t \in[0, T), \\
& \mu(\xi, t)=2 S K\left(\xi, \Gamma_{t}\right)-G_{2,0}(\xi) \quad \text { on } \quad \Gamma_{t}, \quad \partial_{\nu} \mu(\cdot, t)=0 \quad \text { on } \partial \Omega, \quad t \in[0, T), \\
& V_{0}\left(\cdot, \Gamma_{t}\right)=\frac{1}{2}\left[\partial_{\nu} \mu\right]_{\Gamma_{t}}(\xi), \quad \xi \in \Gamma_{t}, \quad t \in(0, T), \\
& \Gamma_{0}=\Gamma^{0},
\end{aligned}
$$

where $G_{1,0}(\xi):=\lim _{\varepsilon \rightarrow 0} G_{1}(\xi ; \varepsilon)$ and $G_{2,0}(\xi):=\lim _{\varepsilon \rightarrow 0} G_{2}(\xi ; \varepsilon)$ and $S>0$ is the surface tension defined in (4.32). In [7] the authors applied formal asymptotics to analyze the sharp interface motion for generalized Cahn-Hilliard equations of the form (1.8). The limit problem, which was formally derived in [7], agrees exactly to (1.18) which is rigorously proven in this paper.

We immediately obtain for any $t \in(0, T)$

$$
2 \int_{\Gamma_{t}} V_{0}\left(\eta, \Gamma_{t}\right) \mathrm{d} S_{\eta}=\int_{\Gamma_{t}}\left[\partial_{\nu} \mu\right]_{\Gamma_{t}}(\eta) \mathrm{d} S_{\eta}=-\int_{\Omega \backslash \Gamma_{t}} \Delta \mu(\eta, t) \mathrm{d} \eta=\int_{\Omega} G_{1,0}(\eta) \mathrm{d} \eta,
$$

i.e., (1.17). Recalling that $\frac{\mathrm{d}}{\mathrm{d} t}\left|\Omega_{\Gamma_{t}}^{-}\right|=\int_{\Gamma_{t}} V_{0}\left(\eta, \Gamma_{t}\right) \mathrm{d} S_{\eta}$, we obtain that the area enclosed by $\Gamma_{t}$ is not conserved unless $\int_{\Omega} G_{1,0}(\eta) \mathrm{d} \eta=0$. Also, we have

$$
\begin{aligned}
\frac{\mathrm{d}}{\mathrm{d} t}\left|\Gamma_{t}\right| & =\int_{\Gamma_{t}} K\left(\eta, \Gamma_{t}\right) V_{0}\left(\eta, \Gamma_{t}\right) \mathrm{d} S_{\eta} \\
& =\frac{1}{2 S}\left(\int_{\Gamma_{t}} \mu V_{0}\left(\eta, \Gamma_{t}\right) \mathrm{d} S_{\eta}+\int_{\Gamma_{t}} V_{0}\left(\eta, \Gamma_{t}\right) G_{2,0}(\eta) \mathrm{d} S_{\eta}\right) .
\end{aligned}
$$


Let us denote by $\mu^{ \pm}\left(\cdot, \Gamma_{t}\right)$ the restriction of $\mu\left(\cdot, \Gamma_{t}\right)$ in $\Omega_{t}^{ \pm}$. It follows that

$$
\begin{aligned}
2 \int_{\Gamma_{t}} \mu V_{0}\left(\eta, \Gamma_{t}\right) \mathrm{d} S_{\eta} & =\int_{\Gamma_{t}} \mu^{+} \partial_{\nu} \mu^{+} \mathrm{d} S_{\eta}-\int_{\Gamma_{t}} \mu^{-} \partial_{\nu} \mu^{-} \mathrm{d} S_{\eta} \\
& =-\int_{\Omega_{t}^{-}} \operatorname{div}(\mu \nabla \mu) \mathrm{d} \xi-\int_{\Omega_{t}^{+}} \operatorname{div}(\mu \nabla \mu) \mathrm{d} \xi \\
& =-\int_{\Omega}|\nabla \mu|^{2} \mathrm{~d} \xi-\int_{\Omega \backslash \Gamma_{t}} \mu \Delta \mu \mathrm{d} \xi=-\int_{\Omega}|\nabla \mu|^{2} \mathrm{~d} \xi+\int_{\Omega \backslash \Gamma_{t}} \mu G_{1,0}(\xi) \mathrm{d} \xi .
\end{aligned}
$$

From these computations there is no reason to expect that $\frac{\mathrm{d}}{\mathrm{d} t}\left|\Gamma_{t}\right|$ is not positive. So, even in the case when the volume is conserved, i.e when $\int_{\Omega} G_{1,0}(\eta) \mathrm{d} \eta=0$ the length of the curve does not decrease. The unknown $\left\{\Gamma_{t} \in \Omega: t \in[0, T]\right\}$ and $\mu^{ \pm}$are coupled through the system (1.18). However if the position and the regularity of the moving boundary $\left\{\Gamma_{t} \in \Omega: t \in[0, T]\right\}$ is known, the chemical potential $\mu$ is obtained by solving at each time $t \in[0, T)$ the elliptic boundary value problem

$$
\begin{aligned}
& \Delta \mu(\xi, t)=-G_{1,0}(\xi), \quad \xi \in \Omega \backslash \Gamma_{t}, \quad t \in[0, T), \\
& \mu(\xi, t)=2 S K\left(\xi, \Gamma_{t}\right)-G_{2,0}(\xi) \quad \text { on } \quad \Gamma_{t}, \quad \partial_{\nu} \mu(\cdot, t)=0 \quad \text { on } \quad \partial \Omega, \quad t \in[0, T) .
\end{aligned}
$$

In this sense we call a family $\{\Gamma(t) ; t \in[0, T)\}$ of surfaces a solution of (1.18). To our knowledge there are no results regarding the existence and the uniqueness of solution for the moving boundary problem of the type (1.18). A modified Mullins Sekerka motion has been studied by [20], but it differs from (1.18) both at the presence of the term $-G_{1,0}$ which is replaced in [20] by a specific function of time only, and might at the presence of $-G_{2,0}$ which does not appear in [20]. We think that a method similar to the one used in [20] might be useful to give existence and uniqueness of the classical solution of (1.18). For the purposes of this paper, we assume that there exists a unique classical solution of the free boundary problem (1.18).

\subsection{Background literature}

There is a large body of literature concerning Cahn-Hilliard equations. We refer the reader to the nice introduction of Novick-Cohen on this subject and to the list of reference therein, see [29]. We also refer to [34] for a connection with the Mullins-Sekerka limit motion in spherical symmetry.

There is also a large number of papers, more physics oriented, devoted to the derivation of generalized Cahn-Hilliard equations and conserved phase field system, see for example [8], [9]. In [10] an interesting discussion of the asymptotic analysis of the phase field equations in the case of different sharp interface limits takes place.

Various generalizations of conserved phase field models are studied in [30], [31], [32]. In the aforementioned papers, the authors consider systems of equations close to our model and take into account more complicated structures such as anisotropy and temperature fields coupled with density, and, derive interesting formal asymptotic expansions of the order parameters (density and temperature) as the scaling parameter $\varepsilon \rightarrow 0$. These systems of equations are more complicated than (1.8), and challenging to analyze.

As a first step for a rigorous analysis of generalized phase field models, in this paper, we set ourself in a simpler context, yet not only proving rigorously that the limit interfacial motion is given 
by (1.18) but also employing a method that allows for the derivation of higher order corrections to the limiting motion.

\section{Notations and main results}

\subsection{Vector fields and flow on the curve space}

Let $m$ denote the set of all smooth simple closed curves in $\Omega \subset \mathbb{R}^{2}$. To discuss motion in $m$ it is convenient to introduce local coordinates in the neighborhood of any given $\Gamma \in \mathcal{m}$. To this aim we define:

Definition 2.1 Let $K(\xi)=K(\xi, \Gamma)$ denote the curvature at a point $\xi \in \Gamma$ for $\Gamma \in \mathcal{M}$. We define

$$
k(\Gamma):=\max _{\xi \in \Gamma}|K(\xi)| .
$$

We denote by $d(\xi, \Gamma)$ the signed distance of $\xi \in \Omega$ from $\Gamma$. We define $d<0$ when $\xi$ is inside $\Gamma$ and $d>0$ when $\xi$ is outside $\Gamma$. As long as $d(\xi, \Gamma) \leqslant \frac{1}{k(\Gamma)}$ there is a uniquely determined point $\eta \in \Gamma$ such that $|\eta-\xi|=d(\xi, \Gamma)$; this is the point in $\Gamma$ closest to $\xi$. Therefore, for any $\varepsilon_{0}$ such that $0<\varepsilon_{0}<\frac{1}{k(\Gamma)}$, let

$$
\eta\left(\varepsilon_{0}\right)=n\left(\varepsilon_{0}, \Gamma\right):=\left\{\xi \in \Omega:|d(\xi, \Gamma)| \leqslant \varepsilon_{0}\right\} .
$$

There is a natural set of coordinates in $\eta\left(\varepsilon_{0}\right)$. Given $\xi \in \eta\left(\varepsilon_{0}\right)$ we denote by $\rho$ the diffeomorphism $\rho: \eta\left(\varepsilon_{0}\right) \rightarrow\left[-\varepsilon_{0}, \varepsilon_{0}\right] \times \Gamma$ defined by $\rho(\xi)=(d(\xi), s(\xi)$ ) (whenever this does not cause ambiguity we omit to write the explicit dependence of $\eta$ or $d$ on $\Gamma$ ). We have that

$$
\xi=s(\xi)+d v(s(\xi))
$$

where $v(s(\xi))$ denotes the unit outward normal to $\Gamma$ at $s(\xi)$. For $d \in\left[-\varepsilon_{0}, \varepsilon_{0}\right]$ and $s \in \Gamma$ let $\alpha(d, s)$ be the Jacobian of the local change of variables $\alpha(d, s)=\operatorname{det} \frac{\partial \rho^{-1}(d, s)}{\partial(d, s)}$. A standard computation (cf. [24, appendix]) gives $\alpha(d, s)=\prod_{i=1}^{n-1}\left(1-d K_{i}(s)\right)$, where $K_{i}(s), i=1, \ldots, n$ are the principal curvatures at $s \in \Gamma$, in the direction $i$. When $n=2$ we have

$$
\alpha(d, s)=1-d K(s) .
$$

In the sequel we identify functions of variable $\xi$ and functions of variable $(d, s)$ in the domain $n\left(\varepsilon_{0}\right)$. We denote by

$$
z=\frac{d}{\varepsilon}
$$

the stretched variable.

The introduced coordinates on $\eta\left(\varepsilon_{0}, \Gamma\right)$ provide the means to give $m$ the structure of a differentiable manifold and to study motions in this manifold, see [14, Section 2]. A vector field $V$ on $m$ is a functional associating to each $\Gamma$ in $m$ a function in $C^{\infty}(\Gamma)$. This function defines the normal velocity of a point on $\Gamma$ and thus, describes a "flow" on $m$. More specifically, we may formally write

$$
\frac{\mathrm{d}}{\mathrm{d} t} \Gamma_{t}=V\left(\Gamma_{t}\right)
$$


We denote the lifetime $T$ of the flow (2.2), starting at $\Gamma \in \mathcal{M}$ as

$$
T=\inf \left\{t>0: k\left(\Gamma_{t}\right) \leqslant k_{0}\right\},
$$

where $k_{0}$ is any arbitrarily chosen positive number so that $k(\Gamma) \leqslant k_{0}<\infty$. If $V(\cdot, \Gamma)=K(\cdot, \Gamma)$, the curvature at $s \in \Gamma$, one obtains the curve shortening flow by curvature. When $V(\cdot, \Gamma)$ is given by (1.7) we have the Mullins-Sekerka vector field, described in the introduction. When $V(\cdot, \Gamma)$ is given by (1.16) we have the flow characterizing the sharp interface motion studied in this paper.

A given flow on $m$ produces an evolution in $C^{\infty}(\Omega)$ through the following device: Let $m$ be any function from $m$ to $C^{\infty}(\Omega)$; we write $m(\xi, \Gamma)$ to denote $m(\Gamma)$ evaluated at $\xi \in \Omega$. Then a time dependent function $m(\xi, t)$ may be defined on $\Omega$ as follows:

$$
m(\xi, t):=m\left(\xi, \Gamma_{t}\right)
$$

There is an obvious but useful decomposition of vector fields on $m$. Given a vector field $V$ on m we may apply the decomposition

$$
V(\cdot, \Gamma)=V^{(0)}(\cdot, \Gamma)+\langle V\rangle_{\Gamma},
$$

where

$$
\langle V\rangle_{\Gamma}:=\frac{1}{|\Gamma|} \int_{\Gamma} V(\xi, \Gamma) \mathrm{d} S_{\xi}
$$

and

$$
V^{(0)}(\cdot, \Gamma):=V(\cdot, \Gamma)-\langle V\rangle_{\Gamma} .
$$

Since $\langle V\rangle_{\Gamma}$ is constant then by its definition $V^{(0)}$ is orthogonal to the constants in the $L^{2}(\Gamma)$ inner product i.e., satisfies

$$
\int_{\Gamma} V^{(0)}(\eta, \Gamma) \mathrm{d} S_{\eta}=0,
$$

and therefore, it generates a volume preserving flow in the sense that for any $t$ the area enclosed by $\Gamma=\Gamma_{t}$ is constant.

Under the ansatz given below, in this paper, we derive separate equations for the components $V^{(0)}(\cdot, \Gamma)$ and $\langle V\rangle_{\Gamma}$ for each of the vector fields $V_{j}$.

ANSATZ 2.2 Let $V_{0}, V_{1}, V_{2}, \cdots$ be a sequence of vector fields on $m$ and $m_{0}, m_{1}, m_{2}, \cdots$ functions from $m$ to $C^{\infty}(\Omega)$. For any given initial interface $\Gamma_{0}$ in $m$ and all $N>0$, let $\Gamma_{t}^{(N)}$ be the solution of

$$
\frac{\mathrm{d} \Gamma_{t}^{(N)}}{\mathrm{d} t}=\left[\sum_{j=0}^{N-1} \varepsilon^{j} V_{j}\right]\left(\Gamma_{t}^{(N)}\right) \quad \text { with } \quad \Gamma_{0}^{(N)}=\Gamma_{0} .
$$

We define the function $m^{(N)}(\xi, t)$ by

$$
m^{(N)}(\xi, t)=m_{0}\left(\frac{d\left(\xi, \Gamma_{t}^{(N)}\right)}{\varepsilon}\right)+\sum_{j=1}^{N} \varepsilon^{j} m_{j}\left(\xi, \Gamma_{t}^{(N)}\right),
$$

and notice that $m^{(N)}(\xi, t)$ depends on $t$ only through $\Gamma_{t}^{(N)}$. 
We set

$$
m_{0}(z):=r\left(\frac{\varepsilon}{\varepsilon_{0}} z\right) \bar{m}(z)+\left(1-r\left(\frac{\varepsilon}{\varepsilon_{0}} z\right)\right) \operatorname{sgn}(z)
$$

where $\bar{m}(z):=\tanh (z / \sqrt{2})^{1}$ defined for any $z \in \mathbb{R}$ is the unique solution of the Euler-Lagrange equation

$$
-m^{\prime \prime}(z)+f(m(z))=0, \quad z \in \mathbb{R}, \quad \lim _{z \rightarrow \pm \infty} m(z)= \pm 1,
$$

and $r$ is a smooth even unimodal cut-off function, $r(u)=1$ for $|u|<\frac{1}{2}$ and $r(u)=0$ for $u>1$.

In addition, let

$$
m_{j}\left(\xi, \Gamma_{t}^{(N)}\right):=h_{j}\left(\frac{d\left(\xi, \Gamma_{t}^{(N)}\right)}{\varepsilon}, s\left(\xi, \Gamma_{t}^{(N)}\right)\right)+\phi_{j}\left(\xi, \Gamma_{t}^{(N)}\right), \quad \xi \in \Omega, \quad j=1, \cdots, N,
$$

where $h_{j}$ are $C^{\infty}(\Omega)$ functions equal to 0 in $\Omega \backslash \eta\left(\varepsilon_{0}\right)$ and when $d\left(\xi, \Gamma_{t}^{(N)}\right)=0$. The functions $\phi_{j}, j=1, \cdots, N$ are in $C^{\infty}(\Omega)$, satisfy the Neumann boundary conditions on $\partial \Omega$ and admit a global Lipschitz bound, independent of $\varepsilon$, i.e.,

$$
\left\|\phi_{j}\right\|_{L i p(\Omega)} \leqslant C, \quad j=1, \cdots, N,
$$

where $C$ is a constant independent of $\varepsilon$.

Notational convention. Below we denote by $m^{(N)}(\xi, t):=m^{(N)}\left(\xi, \Gamma_{t}^{(N)}\right)$ and $\mu^{(N-1)}(\xi, t):=$ $\mu^{(N-1)}\left(\xi, \Gamma_{t}^{(N)}\right)$. If there is no ambiguity we write $\Gamma$ or $\Gamma_{t}$ for $\Gamma_{t}^{(N)}$. In what follows, we write $C$ to designate a generic positive constant independent on $\varepsilon$. Its actual numerical value may change from one occurrence to the next.

Remark 2.3 The Ansatz 2.2 must be modified when $G_{1}$ and $G_{2}$ depend on time. The (2.8) should be replaced by

$$
m^{(N)}(\xi, t)=m_{0}\left(\frac{d\left(\xi, \Gamma_{t}^{(N)}\right)}{\varepsilon}\right)+\sum_{j=1}^{N} \varepsilon^{j} m_{j}\left(\xi, t, \Gamma_{t}^{(N)}\right)
$$

Notice that $m^{(N)}(\xi, t)$ depends now on $t$ not only through $\Gamma_{t}^{(N)}$. One can verify that the first order function $m_{0}$ keeps to depend on $t$ only trough $\Gamma_{t}^{(N)}$.

\subsection{Main results}

We start constructing a function $m^{(N)}\left(\xi, \Gamma_{t}\right)$, for $\xi \in \Omega$ and $t \in[0, T]$ where $T$ is the lifetime of (2.7) and show that it is an approximate solution of (1.8). We make the following assumptions on the forcing terms $G_{1}$ and $G_{2}$.

\footnotetext{
1 The explicit form of the solution is never used. We will use only its qualitative properties.
} 
A1: Assumptions for $G_{1}$ and $G_{2}$.

For any $N>1$ we require

$$
\begin{aligned}
& G_{i}(\xi ; \varepsilon)=\sum_{j=0}^{N-1} \varepsilon^{j} G_{i, j}(\xi)+\varepsilon^{N} G_{i, N}(\xi, \varepsilon), \quad\left|G_{i, N}(\xi, \varepsilon)\right| \leqslant C, \\
& G_{i, j} \in C^{\infty}(\Omega), \quad j=1, \cdots, N-1, \quad i=1,2, \\
& \partial_{\nu} G_{2, j}=0 \quad \text { on } \quad \partial \Omega, \quad j=1, \cdots, N .
\end{aligned}
$$

REMARK 2.4 We require $G_{i, j} \in C^{\infty}(\Omega)$ for $j=1, \cdots, N-1$ and for $i=1,2$ to avoid regularity problems, but this assumption can be relaxed.

Theorem 2.5 Let $N>1$ and $G_{1}$ and $G_{2}$ be as in assumptions A1. There exist vector fields $V_{j}$, $j=0, \cdots,(N-1)$ and functions $m_{j}, j=0, \cdots, N$ as prescribed in the Ansatz 2.2 having the following properties: Let $T$ denote the lifetime of the solution of (2.7) in $m$. Then there is a constant $C_{N}$ so that for all $t<T$

$$
\begin{aligned}
\partial_{t} m^{(N)}(\xi, t)=\Delta\left(-\varepsilon \Delta m^{(N)}(\xi, t)+\frac{1}{\varepsilon} f\left(m^{(N)}(\xi, t)\right)\right. & \left.-\sum_{j=0}^{N-1} \varepsilon^{j} G_{2, j}(\xi)\right) \\
& +\sum_{j=0}^{N-1} \varepsilon^{j} G_{1, j}(\xi)+\Delta R^{(N)}(\xi, t),
\end{aligned}
$$

where

$$
\sup _{\xi \in \Omega, t \in[0, T]}\left|R^{(N)}(\xi, t)\right| \leqslant C_{N} \varepsilon^{N-1} .
$$

Finally, the sequences of vector fields and functions are essentially uniquely determined since given $V_{j}$ for $j<k$ then $V_{k}$ is determined up to $O\left(\varepsilon^{k+1}\right)$, and similarly, given $m_{j}$ for $j<k$ then $m_{k}$ is determined up to $\mathcal{O}\left(\varepsilon^{k+1}\right)$.

REMARK 2.6 In Theorem 2.5 and in the following, the symbol $\mathcal{O}\left(\varepsilon^{m}\right)$ denotes terms which are of order $\varepsilon^{m}$ uniformly in all their variables. The qualified nature of uniqueness stated in this theorem is an indication that there will be choices to be made at every stage of the approximation.

The proof of Theorem 2.5 follows the main lines of the scheme introduced in [14] and it is proven in Section 5. There, the complete result relating the solution of (2.12) and its sharp interface limit is given. The construction behind the proof is patterned on the Hilbert expansion of kinetic theory. We refer the interested reader to [14, Section 3.2] where this connection is discussed. We first construct an approximate solution up to order $N$ of the chemical potential $\mu^{\varepsilon}$ (cf. (1.10)) assuming that the left hand side of (1.10) is known and it is given by the Ansatz 2.2. This is done in Section 3. We, then, insert the constructed approximate chemical potential into (1.11). The approximate solution $m^{(N)}$ is determined provided certain compatibility conditions are verified. This is done in Section 4. Finally in Section 5 we construct $\left(\tilde{m}^{(N)}, \tilde{\mu}^{(N-1)}\right)$ where $\tilde{m}^{(N)}$ is an $\varepsilon^{N}$ modification of $m^{(N)}$ and $\tilde{\mu}^{(N-1)}$ is an $\varepsilon^{N-1}$ modification of $\mu^{(N-1)}$ and we show Theorem 2.5.

Let $V_{j}, j=1, \cdots, N-1$, be the sequence of vector fields introduced in the Ansatz 2.2. According to (2.5), we split them as

$$
V_{j}(\cdot, \Gamma)=V_{j}^{(0)}(\cdot, \Gamma)+\left\langle V_{j}\right\rangle_{\Gamma}, \quad j=1, \cdots, N-1 .
$$


The term $V_{j}^{(0)}$ is determined in Theorem 4.1 by applying the Dirichelet-Neumann operator in the context of potential theory, while the term $\left\langle V_{j}\right\rangle_{\Gamma}$, which is constant on $\Gamma$, is determined in Theorem 3.1.

As already explained in the introduction, the leading term $V_{0}$ in the vector field $\sum_{j=0}^{N-1} \varepsilon^{j} V_{j}\left(\Gamma_{t}^{(N)}\right)$ governing the interfacial flow (cf. (2.7)) is given by

$$
V_{0}\left(\cdot, \Gamma_{t}\right)=V_{0}^{(0)}\left(\cdot, \Gamma_{t}\right)+\left\langle V_{0}\right\rangle_{\Gamma_{t}} .
$$

The family of curves $\left\{\Gamma_{t} \in \mathcal{M}, t \in[0, T]\right\}$ driven by $V_{0}$ is the solution of the moving boundary problem (1.18). For a curve $\Gamma \in \boldsymbol{m}$, the term $V_{0}^{(0)}(\Gamma)$ is determined, see Lemma 4.5 as a real valued function on $\Gamma$ given by

$$
V_{0}^{(0)}(\xi, \Gamma)=\left[\partial_{\nu} \mu_{0,0,0}\right]_{\Gamma}(\xi), \quad \xi \in \Gamma,
$$

where the brackets on the right denote the jump in the normal derivative across $\Gamma$. For each $\Gamma \in \mathcal{M}$, $\mu_{0,0,0}$ is the solution of

$$
\Delta \mu(\xi)=0 \quad \text { for } \quad \xi \in \Omega \backslash \Gamma
$$

subject to the boundary conditions

$$
\begin{array}{r}
\mu(\xi)=S\left(K(\xi)-\frac{\int_{\Gamma} K(\xi) \mathrm{d} S_{\xi}}{|\Gamma|}\right)+\frac{1}{4}\left(B_{0,0,0}(\xi)-\frac{\int_{\Gamma} B_{0,0,0}(\xi) \mathrm{d} S_{\xi}}{|\Gamma|}\right) \\
\text { on } \Gamma, \partial_{\nu} \mu=0 \text { on } \partial \Omega .
\end{array}
$$

The term $B_{0,0,0}$ (cf. (4.39)) is given by

$$
B_{0,0,0}(\xi)=-2\left[\tilde{\mu}_{0,0,0}(\xi)+G_{2,0}(\xi)\right], \quad \xi \in \Gamma,
$$

where (cf. (4.38))

$$
\tilde{\mu}_{0,0,0}(\xi)=\left\langle V_{0}\right\rangle_{\Gamma} 2 \int_{\Gamma} G(\xi, \eta) \mathrm{d} S_{\eta}-\int_{\Omega} G(\xi, \eta) G_{1,0}(\eta) \mathrm{d} \eta,
$$

for $G(\xi, \eta)$ the Green function in $\Omega$, with Neumann boundary condition on $\partial \Omega$, satisfying the equation

$$
\Delta G(\xi, \eta)=\delta(\xi-\eta)-\frac{1}{|\Omega|},
$$

so that

$$
\int_{\Omega} G(\xi, \eta) d \eta=\int_{\Omega} G(\xi, \eta) d \xi=0 .
$$

The second step is to show that there is an actual solution of (1.8) close to the constructed approximate solution $m^{(N)}(\cdot, \cdot)$ whenever both of them start from the initial datum $m_{0}^{\varepsilon}$, see (1.12). This step for the standard Cahn-Hilliard equation (i.e. for $G_{1}=G_{2}=0$ ) has been proved in the work of Alikakos, Bates and Chen, [1], by application of spectral estimates. We use the spectral estimates as in [1]. Namely the linear operator

$$
\mathcal{L} w=\Delta\left(\varepsilon \Delta w-\frac{1}{\varepsilon} f^{\prime}\left(m^{(N)}(t)\right) w\right),
$$


that one obtains linearizing $(1.8)$ at $m^{(N)}(t), t \in[0, T]$; the solution constructed in Theorem 2.5, is the same as in [1]. The approximate solution has the requirements needed to apply the spectral estimates proven by [2] in two space dimensions and by [15] in arbitrary space dimensions and in more general setting. The assumptions imposed on the forcing terms $G_{1}$ and $G_{2}$ together with the assumption (2.21) are enough to have these terms under control. We state the theorem and we outline in the appendix the proof.

Let $p>0$ and $\|\cdot\|_{p, \Omega}$ be the usual norm in $L^{p}(\Omega)$, then for $T>0$ we define the norm

$$
\|u\|_{p, \Omega_{T}}:=\left(\int_{0}^{T}\|u\|_{p, \Omega}^{p} d t\right)^{1 / p} .
$$

Theorem 2.7 Take $N>1, G_{1}$ and $G_{2}$ as in Assumption A1. Further assume that

$$
\int_{\Omega} G_{1, N}(\xi, \varepsilon) d \xi=0, \quad \forall \varepsilon>0
$$

Let $m^{(N)}(t)$ for $t \in[0, T]$ where $T$ is the lifetime of (2.7), be the solution of (2.12). Let $m^{\varepsilon}$ be the solution of the generalized Cahn-Hilliard equation (1.8) supplemented by the boundary conditions (1.14) and having initial datum $m^{\varepsilon}(\xi, 0)=m^{(N)}(\xi, 0), \xi \in \Omega$. Then, there exists $\varepsilon_{0}>0$ so that for all $\varepsilon \in\left(0, \varepsilon_{0}\right]$, for any pair $\lambda>\frac{13}{3}, N>\frac{3 \lambda+5}{3}$, it holds that

$$
\left\|m^{\varepsilon}-m^{(N)}\right\|_{3, \Omega_{T}} \leqslant \varepsilon^{\lambda} .
$$

REMARK 2.8 The result of the previous theorem coincides with the analogous result in $L^{p}\left(\Omega_{T}\right)$ norm of Theorem 2.1 of [1] for the case $G_{1}=G_{2}=0$ in dimensions $n=2$, since $3=p=2 \frac{n+4}{n+2}$ and since $\lambda>\frac{13}{3}=(n+2) \frac{n^{2}+6 n+10}{4 n+16}$.

REMARK 2.9 Set $\tilde{G}_{1, N-1}=G_{1, N-1}+\varepsilon G_{1, N}$ and therefore $\tilde{G}_{1, N}=0$. Determine the velocity field $V_{N-1}$ replacing $G_{1, N-1}$ with $\tilde{G}_{1, N-1}$. In this way the condition (2.21) of Theorem 2.7 is trivially satisfied.

\section{The construction of the approximate chemical potential}

In this section, we apply potential theory to show the following result.

Theorem 3.1 Take $N>1$ and $G_{1}$ as in Assumption A1. Let $\Gamma_{t}^{(N)}, t \in[0, T]$, be the solution of (2.7) in $m$, with $T$ its lifetime, see (2.3). Let $m^{(N)}\left(\cdot, \Gamma_{t}^{(N)}\right)$ be as in the Ansatz 2.2. There is a unique way to determine the $\left\langle V_{j}\right\rangle\left(\Gamma_{t}^{(N)}\right), j=0, \cdots,(N-1)$, such that there exists a unique (up to a constant in $\xi$ ) expansion

$$
\mu^{(N-1)}(\xi, t)=\sum_{i=0}^{N-1} \varepsilon^{i} \mu_{i}(\xi, t) \quad \text { in } \Omega \times(0, T),
$$

with

$$
\partial_{t} m^{(N)}(\xi, t)=\Delta \mu^{(N-1)}(\xi, t)+\sum_{j=0}^{N-1} \varepsilon^{j} G_{1, j}(\xi)+R_{1}(\xi, t, \varepsilon) \quad \text { in } \Omega \times(0, T),
$$


with $R_{1}$ given in (3.14). Further $\mu^{(N-1)}(\cdot, t)$, for $t \in(0, T)$, is a $C^{\infty}(\Omega)$ function satisfying the Neumann homogeneous boundary conditions on $\partial \Omega$,

$$
\sup _{\xi, t \in \Omega \times[0, T]}\left|R_{1}(\xi, t, \varepsilon)\right| \leqslant C(T) \varepsilon^{N-1},
$$

and

$$
\sup _{t \in[0, T]}\left|\int_{\Omega} R_{1}(\xi, t, \varepsilon) d \xi\right| \leqslant C(T) \varepsilon^{N},
$$

where $C(T)$ is a constant independent of $\varepsilon$. Moreover, the terms $\mu_{i}$ appearing in (3.1) are specified by (3.18), (3.27) and (3.38) below.

We look for a function $\mu^{(N-1)}$ from $m$ to $C^{\infty}(\Omega)$ having the form

$$
\mu^{(N-1)}(\xi, \Gamma)=\sum_{i=0}^{N-1} \varepsilon^{i} \mu_{i}(\xi, \Gamma), \quad \xi \in \Omega, \quad \partial_{\nu} \mu_{i}=0 \quad \text { on } \quad \partial \Omega,
$$

where $\mu_{i}, i=0, \cdots, N-1$ are functions to be determined. We insert into (1.10) the function $m^{(N)}$, given by the Ansatz 2.2, and $\mu^{(N-1)}$ given by (3.5), where both are evaluated at $\Gamma:=\Gamma_{t}^{(N)}$, for $\Gamma_{t}^{(N)}$ the solution of (2.7). Therefore, we obtain $(N-1)$ Laplace equations for $\mu_{i}\left(\cdot, \Gamma_{t}^{(N)}\right)$, $i=1, \cdots,(N-1)$. The compatibility conditions are needed in order to solve these equations and determine $\left\langle V_{j}\right\rangle\left(\Gamma_{t}^{(N)}\right)$ for $j=0, \cdots,(N-1)$.

When differentiating $m^{(N)}\left(\cdot, \Gamma_{t}^{(N)}\right)$ with respect to $t$ we need to take into account that $m^{(N)}$ depends on $\Gamma_{t}$ through a fast and slow scale. The fast scale brings a factor $\varepsilon^{-1}$.

DEFinition 3.2 Let $m$ be a function from $m$ to $C^{\infty}(\Omega)$ of the type $h\left(\frac{d(\xi, \Gamma)}{\varepsilon}, s(\xi, \Gamma)\right)$ and $V$ be a vector field on $\mathrm{m}$. We define

$$
D_{V} m(\xi, \Gamma):=\frac{1}{\varepsilon} h^{\prime}\left(\frac{d(\xi, \Gamma)}{\varepsilon}, s(\xi, \Gamma)\right) V(s(\xi)),
$$

where the prime indicates the derivative of $h$ with respect to the first variable $z=\frac{d(\xi, \Gamma)}{\varepsilon}$.

In addition, for any $W_{N}:=\sum_{j=0}^{N-1} \varepsilon^{j} V_{j}$ with $V_{0}, \cdots, V_{N-1}$ vector fields on $m$, we define

$$
D_{W_{N}} m(\xi, \Gamma):=\sum_{j=0}^{N-1} \varepsilon^{j} D_{V_{j}} m(\xi, \Gamma) .
$$

Note that by the orthogonality of $\nabla_{\xi} d$ with respect to the surface there is no contribution in (3.6) from $s(\xi, \Gamma)$. Therefore, cf. [14] for the detailed computations, differentiating (2.8) with respect to $t$, applying the chain rule at the right-hand side (here the velocity will appear since $m_{j}$ are defined on $\Gamma$ ) and then using (2.10), (3.6) and (3.7), we arrive at

$$
\begin{aligned}
& \partial_{t} m^{(N)}=D_{W_{N}}\left(m^{(N)}\right)=D_{V_{0}} m_{0}+\varepsilon\left[D_{V_{1}} m_{0}+D_{V_{0}} m_{1}\right] \\
& \quad+\varepsilon^{2}\left[D_{V_{1}} m_{1}+D_{V_{0}} m_{2}+D_{V_{2}} m_{0}\right]+\cdots+\varepsilon^{N-1}\left[\sum_{i=0}^{N-1} D_{V_{i}} m_{N-1-i}\right]+R_{N}+E,
\end{aligned}
$$


where

$$
R_{N} \equiv \varepsilon^{N}\left[\sum_{i=0}^{N-1} D_{V_{i}} m_{N-i}\right]+\circlearrowleft\left(\varepsilon^{N}\right)
$$

The $m_{0}$ is the function defined in (2.9) and the term $E \equiv E(\xi, t, \varepsilon)$ is obtained by differentiating $r\left(\frac{d\left(\xi, \Gamma_{t}\right)}{\varepsilon_{0}}\right)$, the unimodal function appearing in the definition of $m_{0}$, with respect to the velocity field. $E$ is given by

$$
E=\frac{1}{\varepsilon_{0}} r^{\prime}\left(\frac{d\left(\xi, \Gamma_{t}\right)}{\varepsilon_{0}}\right)\left[\sum_{i=0}^{N-1} \varepsilon^{i} V_{i}(\sigma(\xi), t)\right]\left\{\bar{m}-\left[\mathbb{I}_{\left\{d\left(\xi, \Gamma_{t}\right)>0\right\}}-\mathbb{I}_{\left\{d\left(\xi, \Gamma_{t}\right)<0\right\}}\right]\right\} .
$$

Note that $E$ is exponentially small since $r^{\prime}$ is different from zero only for $\frac{\varepsilon_{0}}{2 \varepsilon} \leqslant|z| \leqslant \frac{\varepsilon_{0}}{\varepsilon}$ while $\bar{m}$ converges exponentially fast to \pm 1 as $z \rightarrow \pm \infty$, [14]. Taking into account (3.8) and (1.10) we obtain a set of $N$ equations for the $\mu_{i}, i=0, \cdots, N-1$.

Zero order term in $\varepsilon$ :

$$
\left\{\begin{array}{l}
D_{V_{0}} m_{0} \equiv \frac{1}{\varepsilon} V_{0} m^{\prime}=\Delta \mu_{0}+G_{1,0} \quad \text { for } \quad \xi \in \Omega, \quad t \in[0, T], \\
\partial_{v} \mu_{0}=0 \text { on } \partial \Omega
\end{array}\right.
$$

where $G_{1,0}$ is the zero order term in $\varepsilon$ of $G_{1}$.

First order term in $\varepsilon$ :

$$
\left\{\begin{array}{l}
{\left[D_{V_{1}} m_{0}+D_{V_{0}} m_{1}\right]=\Delta \mu_{1}+G_{1,1} \quad \text { for } \quad \xi \in \Omega, \quad t \in[0, T],} \\
\partial_{\nu} \mu_{1}=0 \text { on } \partial \Omega .
\end{array}\right.
$$

$n$-th order term in $\varepsilon(n \leqslant N-1)$ :

$$
\left\{\begin{array}{l}
{\left[\sum_{i=0}^{n} D_{V_{i}} m_{n-i}\right]=\Delta \mu_{n}+G_{1, n} \text { for } \xi \in \Omega, \quad t \in[0, T],} \\
\partial_{\nu} \mu_{n}=0 \text { on } \partial \Omega .
\end{array}\right.
$$

\section{Remainder term:}

The remainder term, see (3.9) and (3.10), is given by

$$
R_{1}(\xi, t, \varepsilon)=\varepsilon^{N} G_{1, N}(\xi, \varepsilon)+R_{N}(\xi, t, \varepsilon)+E(\xi, t, \varepsilon) .
$$

Since the derivative in $R_{N}$ (cf. (3.9)) brings down a factor $\varepsilon^{-1}$ then this yields easily the next estimate for $R_{1}$

$$
\sup _{(\xi, t) \in \Omega \times[0, T]}\left|R_{1}(\xi, t)\right| \leqslant C(T) \varepsilon^{N-1} .
$$


Further, one gains an extra power of $\varepsilon$ when integrating $R_{1}$, since the terms of order $\varepsilon^{N-1}$ have support in $\eta\left(\varepsilon_{0}\right)$

$$
\sup _{t \in[0, T]} \int_{\Omega}\left|R_{1}(\xi, t, \varepsilon)\right| \mathrm{d} \xi \leqslant C(T) \varepsilon^{N} .
$$

In the sequel, we prove existence and uniqueness (up to a constant) of solutions of the equations obtained so far at different orders. In Lemma 3.3 and in Lemma 3.5 we consider the first and second order equation respectively. Finally, in Lemma 3.6 we outline the proof for solving the equation to a generic order. In the next lemma we write in an explicit way the dependence of the mean velocity on $\varepsilon$. This is done in order to get easily the leading velocity field governing the interfacial flows, see (2.7).

LEMMA 3.3 Under the conditions

$$
\begin{gathered}
V_{0}\left(\cdot, \Gamma_{t}\right)=V_{0}^{(0)}\left(\cdot, \Gamma_{t}\right)+\left\langle V_{0}\right\rangle_{\Gamma_{t}}\left[1+c_{0}(\varepsilon)\right], \\
\int_{\Gamma_{t}} V_{0}^{(0)}\left(\eta, \Gamma_{t}\right) \mathrm{d} S_{\eta}=0, \quad\left\langle V_{0}\right\rangle_{\Gamma_{t}}=\frac{1}{2\left|\Gamma_{t}\right|} \int_{\Omega} G_{1,0}(\eta) \mathrm{d} \eta, \quad t \in[0, T],
\end{gathered}
$$

where $c_{0}(\varepsilon)$ defined in (3.24) goes to zero exponentially fast as $\varepsilon \rightarrow 0$, there exists a unique solution (up to constants in $\xi$ ) of (3.11) given by

$$
\mu_{0}\left(\xi, \Gamma_{t}\right)=\mu_{0,0}\left(\xi, \Gamma_{t}\right)+\tilde{\mu}_{0}\left(\xi, \Gamma_{t}\right),
$$

where

$$
\mu_{0,0}\left(\xi, \Gamma_{t}\right)=\int_{\Omega} G(\xi, \eta)\left(\frac{1}{\varepsilon} m_{0}^{\prime}\left(\frac{d\left(\eta, \Gamma_{t}\right)}{\varepsilon}\right) V_{0}^{(0)}\left(s(\eta), \Gamma_{t}\right)\right) \mathrm{d} \eta+c_{0}(t) .
$$

Here, $c_{0}(t)$ is a constant (in $\xi$ ) to be determined, and

$$
\tilde{\mu}_{0}\left(\xi, \Gamma_{t}\right)=\left\langle V_{0}\right\rangle_{\Gamma_{t}}\left[1+c_{0}(\varepsilon)\right] \int_{\Omega} G(\xi, \eta)\left(\frac{1}{\varepsilon} m_{0}^{\prime}\left(\frac{d\left(\eta, \Gamma_{t}\right)}{\varepsilon}\right)\right) \mathrm{d} \eta-\int_{\Omega} G(\xi, \eta) G_{1,0}(\eta) \mathrm{d} \eta .
$$

The term $\mu_{0}$ is in $C^{\infty}(\Omega)$ for any $t \in[0, T]$.

Proof. Because $\partial_{\nu} \mu_{0}=0$ on $\partial \Omega$, the solvability of (3.11) requires that for all $t \in[0, T]$

$$
\int_{\Omega}\left(\frac{1}{\varepsilon} m_{0}^{\prime}\left(\frac{d\left(\eta, \Gamma_{t}\right)}{\varepsilon}\right) V_{0}(s(\eta), t)\right) \mathrm{d} \eta-\int_{\Omega} G_{1,0}(\eta) \mathrm{d} \eta=0 .
$$

In two dimensions (note that in three dimensions there will be extra terms), by using local coordinates it follows that

$$
\begin{aligned}
& \int_{\Omega}\left(\frac{1}{\varepsilon} m_{0}^{\prime}\left(\frac{d\left(\eta, \Gamma_{t}\right)}{\varepsilon}\right) V_{0}(s(\eta), t)\right) \mathrm{d} \eta=\int_{\eta\left(\varepsilon_{0}\right)}\left(\frac{1}{\varepsilon} m_{0}^{\prime}\left(\frac{d\left(\eta, \Gamma_{t}\right)}{\varepsilon}\right) V_{0}(s(\eta), t)\right) \mathrm{d} \eta \\
& =\frac{1}{\varepsilon} \int_{\Gamma} \int_{-\frac{\varepsilon_{0}}{\varepsilon}}^{\frac{\varepsilon_{0}}{\varepsilon}} m_{0}^{\prime}(z) V_{0}(s, t) \varepsilon(1-\varepsilon z K(s)) \mathrm{d} s \mathrm{~d} z \\
& =\int_{\Gamma} \int_{-\frac{\varepsilon_{0}}{\varepsilon}}^{\frac{\varepsilon_{0}}{\varepsilon}} m_{0}^{\prime}(z) V_{0}(s, t) \mathrm{d} s \mathrm{~d} z-\varepsilon \int_{\Gamma} \int_{-\frac{\varepsilon_{0}}{\varepsilon}}^{\frac{\varepsilon_{0}}{\varepsilon}} z m_{0}^{\prime}(z) K(s) V_{0}(s, t) \mathrm{d} s \mathrm{~d} z \\
& =\int_{\Gamma} \int_{-\frac{\varepsilon_{0}}{\varepsilon}}^{\frac{\varepsilon_{0}}{\varepsilon}} m_{0}^{\prime}(z) V_{0}(s, t) \mathrm{d} s \mathrm{~d} z=2\left(1-e^{-\frac{\varepsilon_{0}}{\varepsilon}}\right) \int_{\Gamma_{t}} V_{0}\left(\eta, \Gamma_{t}\right) \mathrm{d} S_{\eta} .
\end{aligned}
$$


The last line holds true since $m_{0}^{\prime}$ is even and exponentially decreasing. Replacing now (3.22) in (3.21), we obtain

$$
\int_{\Omega} G_{1,0}(\eta) \mathrm{d} \eta=2\left(1-e^{-\frac{\varepsilon_{0}}{\varepsilon}}\right) \int_{\Gamma_{t}} V_{0}\left(\eta, \Gamma_{t}\right) \mathrm{d} S_{\eta}
$$

Taking into account the splitting $V_{0}=V_{0}^{(0)}+\left\langle V_{0}\right\rangle_{\Gamma}\left[1+c_{0}(\varepsilon)\right]$, (cf. the first and second equality in (3.17)), and using (3.23), we arrive at

$$
\int_{\Gamma_{t}} V_{0}\left(\eta, \Gamma_{t}\right) d S_{\eta}=\left|\Gamma_{t}\right|\left\langle V_{0}\right\rangle_{\Gamma}\left[1+c_{0}(\varepsilon)\right]=\frac{1}{2}\left(1+c_{0}(\varepsilon)\right) \int_{\Omega} G_{1,0}(\eta) \mathrm{d} \eta
$$

where

$$
c_{0}(\varepsilon):=\frac{e^{-\frac{\varepsilon_{0}}{\varepsilon}}}{\left(1-e^{-\frac{\varepsilon_{0}}{\varepsilon}}\right)} .
$$

This forces us to take $V_{0}$ satisfying the third relation of (3.17). By potential theory, once the compatibility condition is satisfied, the solution is given by (3.18).

REMARK 3.4 Note that $\left\langle V_{0}\right\rangle_{\Gamma_{t}}$ and $\tilde{\mu}_{0}\left(\xi, \Gamma_{t}\right)$ are completely determined once we know $m_{0}$. The quantity $\mu_{0,0}\left(\xi, \Gamma_{t}\right)$ depends on $c_{0}(t)$ and $V_{0}^{(0)}$. These quantities will be determined when proving Theorem 4.1.

\subsection{The first order term in $\varepsilon$}

For the derivation of the first order correction we need to prove the solvability of equation (3.12).

LEMma 3.5 There exists a unique (up to constants in $\xi$ ) solution $\mu_{1}$ of (3.12) provided that

$$
V_{1}\left(\Gamma_{t}\right) \equiv V_{1}^{(0)}\left(\Gamma_{t}\right)+\left\langle V_{1}\right\rangle_{\Gamma_{t}}
$$

with

$$
\int_{\Gamma_{t}} V_{1}^{(0)}\left(\eta, \Gamma_{t}\right) \mathrm{d} S_{\eta}=0, \quad \forall t \in[0, T],
$$

and $\left\langle V_{1}\right\rangle_{\Gamma_{t}}$ chosen according to

$$
\left\langle V_{1}\right\rangle_{\Gamma_{t}}=\frac{1}{2\left|\Gamma_{t}\right|}\left[1+c_{0}(\varepsilon)\right]\left[\int_{\Omega} G_{1,1}(\xi) d \xi-b_{1}(t)\right],
$$

where $b_{1}(t)$ is defined in (3.30) and $c_{0}(\varepsilon)$ in (3.24). The solution is given by

$$
\mu_{1}(\xi, t)=\mu_{1,0}(\xi, t)+\tilde{\mu}_{1}(\xi, t),
$$

where $\tilde{\mu}_{1}$ is defined in (3.35), while

$$
\mu_{1,0}(\xi, t)=\int_{\Omega} G(\xi, \eta)\left(\frac{1}{\varepsilon} m_{0}^{\prime}\left(\frac{d\left(\eta, \Gamma_{t}\right)}{\varepsilon}\right) V_{1}^{(0)}(s(\eta), t)\right) \mathrm{d} \eta+c_{1}(t)
$$

Here, $c_{1}(t)$ is a constant (in $\xi$ ) to be determined. In addition, the solution is a $C^{\infty}(\Omega)$ function for any $t \in[0, T]$. 
Proof. The solvability of (3.12) requires that

$$
\int_{\Omega}\left[D_{V_{1}} m_{0}+D_{V_{0}} m_{1}\right] d \xi-\int_{\Omega} G_{1,1}(\xi) d \xi=0
$$

for any $t \in[0, T]$. Here, we are assuming that $m_{1}, m_{0}$ and $V_{0}$ are already determined and so we define

$$
b_{1}(t)=\int_{\Omega} D_{V_{0}} m_{1} \mathrm{~d} \xi
$$

Proceeding as in (3.22) we obtain

$$
\int_{\Omega} D_{V_{1}} m_{0} \mathrm{~d} \xi=2\left(1-e^{-\frac{\varepsilon_{0}}{\varepsilon}}\right) \int_{\Gamma_{t}} V_{1}(\eta) \mathrm{d} S_{\eta}
$$

Taking into account the decomposition (3.25) and relation (3.26) we have

$$
\int_{\Gamma_{t}} V_{1}\left(\eta, \Gamma_{t}\right) \mathrm{d} S_{\eta}=\left|\Gamma_{t}\right|\left\langle V_{1}\right\rangle_{\Gamma_{t}} .
$$

Therefore, relation (3.29) is satisfied if

$$
\left\langle V_{1}\right\rangle_{\Gamma_{t}}=\frac{1}{2\left|\Gamma_{t}\right|}\left[1+c_{0}(\varepsilon)\right]\left[\int_{\Omega} G_{1,1}(\xi) d \xi-b_{1}(t)\right],
$$

where $c_{0}(\varepsilon)$ is defined in (3.24). This determines $\left\langle V_{1}\right\rangle_{\Gamma_{t}}$, the projection of $V_{1}\left(\Gamma_{t}\right)$ onto the constants. The solution of (3.12) exists and it is given by

$$
\mu_{1}(\xi, t)=\int_{\Omega} G(\xi, \eta)\left[D_{V_{1}} m_{0}+D_{V_{0}} m_{1}\right] \mathrm{d} \eta-\int_{\Omega} G(\xi, \eta) G_{1,1}(\eta) \mathrm{d} \eta+c_{1}(t) .
$$

Since we shall use the decomposition (3.25), it is convenient to write

$$
\mu_{1}(\xi, t)=\mu_{1,0}(\xi, t)+\tilde{\mu}_{1}(\xi, t),
$$

where $\mu_{1,0}(\xi, t)$ is given in (3.28) and $\tilde{\mu}_{1}$ is defined as follows

$$
\begin{aligned}
& \tilde{\mu}_{1}(\xi, t)=\int_{\Omega} G(\xi, \eta) D_{V_{0}} m_{1} \mathrm{~d} \eta+\left\langle V_{1}\right\rangle_{\Gamma_{t}} \int_{\Omega} G(\xi, \eta)\left(\frac{1}{\varepsilon} m_{0}^{\prime}\left(\frac{d\left(\eta, \Gamma_{t}\right)}{\varepsilon}\right)\right) \mathrm{d} \eta \\
&-\int_{\Omega} G(\xi, \eta) G_{1,1}(\eta) \mathrm{d} \eta .
\end{aligned}
$$

LEMmA 3.6 The solution $\mu_{j}(\cdot, t)$ of (3.13) for $2 \leqslant j \leqslant N-1$ exists and is unique (up to constant in $\xi$ ) provided that

$$
\begin{gathered}
V_{j}\left(\Gamma_{t}\right) \equiv V_{j}^{(0)}\left(\Gamma_{t}\right)+\left\langle V_{j}\right\rangle_{\Gamma_{t}}, \\
\int_{\Gamma} V_{j}^{(0)}\left(s, \Gamma_{t}\right) \mathrm{d} s=0, \quad \forall t \in[0, T],
\end{gathered}
$$


and $\left\langle V_{j}\right\rangle_{\Gamma_{t}}$ is chosen according to (3.44). It is given by

$$
\mu_{j}(\xi, t)=\mu_{j, 0}(\xi, t)+\tilde{\mu}_{j}(\xi, t),
$$

where

$$
\mu_{j, 0}(\xi, t)=\int_{\Omega} G(\xi, \eta)\left(\frac{1}{\varepsilon} m_{0}^{\prime}\left(\frac{d\left(\eta, \Gamma_{t}\right)}{\varepsilon}\right) V_{j}^{(0)}(s(\eta), t)\right) \mathrm{d} \eta+c_{j}(t)
$$

and

$$
\begin{aligned}
\tilde{\mu}_{j}(\xi, t)=\int_{\Omega} G(\xi, \eta)\left[\sum_{n=0}^{j-1} D_{V_{n}} m_{j-n}\right] \mathrm{d} \eta \\
+\left\langle V_{j}\right\rangle_{\Gamma_{t}} \int_{\Omega} G(\xi, \eta)\left(\frac{1}{\varepsilon} m_{0}^{\prime}\left(\frac{d\left(\eta, \Gamma_{t}\right)}{\varepsilon}\right)\right) \mathrm{d} \eta+\int_{\Omega} G(\xi, \eta) G_{1, j}(\eta) \mathrm{d} \eta .
\end{aligned}
$$

The solution $\mu_{j}(\cdot, t)$ for $t \in(0, T]$ is a $C^{\infty}(\Omega)$ function.

Proof. The proof is analogous to the proof of Lemma 3.5. The solution exists if

$$
\int_{\Omega}\left[\sum_{n=0}^{j} D_{V_{n}} m_{j-n}\right] \mathrm{d} \xi-\int_{\Omega} G_{1, j}(\xi) d \xi=0
$$

for any $t \in[0, T]$. Here, $D_{V_{n}} m_{j-n}$ for $n=0, \cdots, j-1$ are determined and so, we define

$$
b_{j}(t)=\int_{\Omega}\left[\sum_{n=0}^{j-1} D_{V_{n}} m_{j-n}\right] \mathrm{d} \xi .
$$

Requiring (3.36) and (3.37) we obtain

$$
\int_{\Omega} D_{V_{j}} m_{0} \mathrm{~d} \xi=2\left|\Gamma_{t}\right|\left[1-e^{-\frac{e_{0}}{\varepsilon}}\right]\left\langle V_{j}\right\rangle_{\Gamma_{t}} .
$$

Hence, to fulfill relation (3.41), we must take

$$
\left\langle V_{j}\right\rangle_{\Gamma_{t}}=\frac{1}{2\left|\Gamma_{t}\right|}\left[1+c_{0}(\varepsilon)\right]\left[\int_{\Omega} G_{1, j}(\xi) d \xi-b_{j}(t)\right],
$$

where $c_{0}(\varepsilon)$ is defined in (3.24). This determines $\left\langle V_{j}\right\rangle_{\Gamma_{t}}$, the projection of $V_{j}\left(\Gamma_{t}\right)$ onto the constants. It still remains to determine the orthogonal part $V_{j}^{(0)}$. The solution of (3.13) exists and is given by (3.38).

\subsection{Proof of Theorem 3.1}

From Lemma 3.3, Lemma 3.5 and Lemma 3.6 we have that $\mu^{(N-1)}$ satisfies by construction (3.2). The remainder $R_{1}$ is defined in (3.14) and estimated in (3.15) and (3.16). The term $\mu^{(N-1)}(\cdot, t)$ for $t \in[0, T]$ satisfies the homogeneous Neumann boundary conditions by construction. Thus, Theorem 3.1 holds true. 


\section{Derivation of the equations for $m^{(N)}$}

The next theorem assures the existence and (essential) uniqueness of the functions $m_{j}, j=$ $0, \cdots, N$, having the properties required in the Ansatz 2.2. Existence and uniqueness are obtained provided that a certain compatibility condition is satisfied. This determines $V_{j}^{(0)}$, the orthogonal part of the velocity field.

TheOREM 4.1 Take $N>1$ and $G_{2}$ as in Assumption A1. Let $T$ be the lifetime of the solution of (2.7) in $m$. Let $\mu^{(N-1)}(\cdot, t), t \in[0, T]$ be the function constructed in Theorem 3.1. Then it is possible to choose the vector fields $V_{j}^{(0)}$ so that there exist $m_{j}, j=0, \cdots, N$, having the properties prescribed in the Ansatz 2.2 such that

$\mu^{(N-1)}(\xi, t)=-\varepsilon \Delta m^{(N)}(\xi, t)+\frac{1}{\varepsilon} f\left(m^{(N)}(\xi, t)\right)-\sum_{j=0}^{N-1} \varepsilon^{j} G_{2, j}(\xi)+R_{2}(\xi, t, \varepsilon) \quad$ in $\quad \Omega \times(0, T]$,

with $R_{2}$ given by (4.71). Further, $m^{(N)}(\cdot, t)$ for $t \in[0, T]$ is a $C^{\infty}(\Omega)$ function that satisfies the homogeneous Neumann boundary conditions and

$$
\sup _{\xi \in \Omega} \sup _{t \in[0, T]}\left|R_{2}(\xi, t, \varepsilon)\right| \leqslant C \varepsilon^{N}
$$

Finally, the choice of the term $V_{j}^{(0)}$ is specified by the equations (4.31), (4.51) and (4.63) given below.

In (1.11) we insert at the left-hand side the already determined function $\mu^{(N-1)}\left(\cdot, \Gamma_{t}^{(N)}\right)$ and we obtain

$$
\mu^{(N-1)}(\xi, t)=-\varepsilon \Delta m(\xi, t)+\frac{f(m(\xi, t))}{\varepsilon}-G_{2}(\xi ; \varepsilon) \quad \text { in } \quad \Omega \times(0, T) .
$$

Then $\mu^{(N-1)}$ is written in terms of $m^{(N)}$, chosen according to the ansatz. Here, we prove that there exists a unique way to find the function $m^{(N)}$, having indeed the property required in the ansatz and satisfying equation (4.3) in a certain sense (to be specified in the sequel).

The existence of any $m_{j}, j=0, \cdots, N$ is obtained provided that a compatibility condition is satisfied. This compatibility condition forces us to define properly $V_{j}^{(0)}, j=0, \cdots,(N-1)$.

We distinguish two main steps:

- Step 1: Determination at any order of the equations. This is carried out in Section 4.1.

- Step 2: Analysis of the equations derived at Step 1. This will be done in the Section 4.2.

In the present and in the next section $\Gamma_{t}$ is kept fixed, therefore, for the sake of a simpler notation we drop the subscript $t$, except of the cases that a subscript use may add some clarity in our arguments.

\subsection{Determination of the equations for $m_{j}, j=0, \cdots N$}

To separate the fast and slow scale of $m^{(N)}$ near the surface $\Gamma$, we write the Laplacian in the system of local coordinates introduced in Section 2.1. The expansion in $\varepsilon$ of the Laplacian written in this coordinate system is reported in the Appendix, Section A.2. We then match the right and left terms of the equations having the same power of $\varepsilon$, distinguishing the case where $\xi \in \eta\left(\varepsilon_{0}\right)$ from the one with $\xi \in \Omega \backslash \eta\left(\varepsilon_{0}\right)$. We therefore, get at any order two sets of equations: one for $\xi \in \eta\left(\varepsilon_{0}\right)$ and the 
other for $\xi \in \Omega \backslash \eta\left(\varepsilon_{0}\right)$. Since the interface separates $\Omega$ in two regions we will distinguish those $\xi \in \Omega \backslash \eta\left(\varepsilon_{0}\right)$ which are inside $\Gamma$ from those $\xi \in \Omega \backslash \eta\left(\varepsilon_{0}\right)$ which are outside $\Gamma$.

Taking into account formula (A.2.2) in the Appendix, denoting by ' the derivative with respect to $z$, and by $a_{n}, b_{n}, c_{n}$ the quantities defined in (A.2.3), after simple, however lengthy computations we obtain the following identity

$$
\begin{aligned}
& \varepsilon^{2} \Delta m^{(N)}(z, s)=\left\{\bar{m}^{\prime \prime}(z)+\sum_{n=1}^{N} \varepsilon^{n}\left[h_{n}^{\prime \prime}(z, s)+a_{n}(z, s) \bar{m}^{\prime}\right]\right\} \\
&+\left\{\sum_{n=2}^{N} \varepsilon^{n} \sum_{i=1}^{n-1} a_{n-i}(z, s) h_{i}^{\prime}(z, s)+\sum_{n=3}^{N} \varepsilon^{n}\left[\sum_{i=1}^{n-2} b_{n-i}(z, s) \frac{d^{2}}{d s^{2}} h_{i}(z, s)\right]\right. \\
&\left.+\sum_{n=4}^{N} \varepsilon^{n} \sum_{i=1}^{n-3} c_{n-i}(z, s) \frac{d}{d s} h_{i}(z, s)\right\}+\varepsilon^{2} \Delta\left[\sum_{i=1}^{N} \varepsilon^{i} \phi_{i}(\xi)\right] \\
&+E_{1}(\xi, t, \varepsilon)+\varepsilon^{N+1} A(\xi, t, \varepsilon),
\end{aligned}
$$

with

$$
\begin{gathered}
\sup _{(\xi, t) \in \Omega \times[0, T]}|A(\xi, t, \varepsilon)| \leqslant C(T), \\
\sup _{t \in[0, T]} \int_{\Omega} d \xi|A(\xi, t, \varepsilon)| \leqslant \varepsilon C(T), \\
E_{1}(\xi, \varepsilon) \equiv \varepsilon^{2} \Delta r\left(\frac{d(\xi, \Gamma)}{\varepsilon_{0}}\right)\left\{\bar{m}\left(\frac{d(\xi, \Gamma)}{\varepsilon}\right)-\left[\mathbb{I}_{\{d(\xi, \Gamma)>0\}}-\mathbb{I}_{\{d(\xi, \Gamma)<0\}}\right]\right\}, \\
+2 \varepsilon^{2} \nabla r \cdot \nabla\left[\bar{m}\left(\frac{d(\xi, \Gamma)}{\varepsilon}\right)\right]
\end{gathered}
$$

and

$$
\lim _{\varepsilon \rightarrow 0} \sup _{(\xi, t) \in \Omega \times[0, T]}\left|E_{1}(\xi, t, \varepsilon)\right|=0 .
$$

Let us now define $f_{i}$ such that

$$
\begin{gathered}
f\left(m^{(N)}\right)=f\left(m_{0}\right)+f^{\prime}\left(m_{0}\right)\left[\sum_{i=1}^{N} \varepsilon^{i} m_{i}\right]+\sum_{i=2}^{N} \varepsilon^{i} f_{i}\left(m_{0}, m_{1}, \ldots, m_{i-1}\right)+\varepsilon^{N+1} B_{N+1}(\cdot, \varepsilon), \\
\sup _{\xi \in \Omega, t \in[0, T]}\left|B_{N+1}(\xi, t, \varepsilon)\right| \leqslant C .
\end{gathered}
$$

One can easily obtain $f_{i}$ for any $i=2, \cdots, N$ by using Taylor expansions up to $N-1$ order for $f$ around $m_{0}$ and collecting then the terms of the same power of $\varepsilon$.

We insert (4.4) and (4.9) into (4.3) and equate terms having the same order in $\varepsilon$ (when estimated with the $L^{\infty}(\Omega)$ norm) obtaining, this way, two equations at any order, one for $\xi \in \Omega \backslash \eta\left(\varepsilon_{0}\right)$, and the other for $\xi \in \eta\left(\varepsilon_{0}\right)$. The equation for $\xi \in \Omega \backslash \eta\left(\varepsilon_{0}\right)$ will determine $\phi_{i}$ which are the slowly varying terms, while the one for $\xi \in \eta\left(\varepsilon_{0}\right)$ will determine $h_{i}$ i.e. the rapidly decaying terms. When 
deriving the equations for $\xi \in \eta\left(\varepsilon_{0}\right)$ then terms of the type $\Delta \phi_{i}(\varepsilon z, s)$ appear. The $\phi_{i}$ are $C^{\infty}$ functions, since they are proportional to $\mu_{i}$, and they have the same type of singularity in $\varepsilon$ when differentiated in $\xi$. So, the terms $\varepsilon^{n+1} \Delta \phi_{n-1}(\varepsilon z, s)$ are of order $\mathcal{O}\left(\varepsilon^{n}\right)$, and therefore, we write them in the $\varepsilon^{n}$ order equation.

In the following we will use the notation $f( \pm 1)$ (or $f(m( \pm \infty))$ ) in $\Omega \backslash \eta\left(\varepsilon_{0}\right)$. This refers to the fact that $\Gamma$ separates $\Omega$ in two sets, i.e. $\Omega^{+}$which is the part outside $\Gamma$ and $\Omega^{-}$the part inside $\Gamma$. Therefore, \pm refers to different regions of $\left(\Omega \backslash \eta\left(\varepsilon_{0}\right)\right) \cap \Omega^{ \pm}$. It is convenient to write the external potential $G_{2, i}$, for $i=0, \cdots, N$ in local coordinates when $\xi \in \eta\left(\varepsilon_{0}\right)$. We therefore identify $G_{2, i}(\xi)=G_{2, i}(d(\xi, \Gamma), s(\xi, \Gamma))$, for $i=0, \cdots, N$.

Zero order term in $\varepsilon$ :

Matching gives

$$
0=-r\left(\frac{\varepsilon}{\varepsilon_{0}} z\right) m_{0}^{\prime \prime}(z)+f\left(m_{0}(z)\right) \quad \text { for } \quad z \in\left[-\frac{\varepsilon_{0}}{\varepsilon}, \frac{\varepsilon_{0}}{\varepsilon}\right] \text {, }
$$

and

$$
f\left(m_{0}( \pm \infty)\right)=0 \quad \text { for } \quad \xi \in \Omega \backslash \eta\left(\varepsilon_{0}\right) .
$$

First order term in $\varepsilon$ :

Again matching gives for $z \in\left[-\frac{\varepsilon_{0}}{\varepsilon}, \frac{\varepsilon_{0}}{\varepsilon}\right]$ and $s \in \Gamma$

$$
\mu_{0}(\varepsilon z, s)=-\left[h_{1}^{\prime \prime}(z, s)-K(s) m_{0}^{\prime}(z)\right]+f^{\prime}\left(m_{0}\right)\left[h_{1}(z, s)+\phi_{1}(\varepsilon z, s)\right]-G_{2,0}(\varepsilon z, s)
$$

and

$$
\mu_{0}(\xi)=f^{\prime}(\bar{m}( \pm \infty)) \phi_{1}(\xi)-G_{2,0}(\xi) \text { for } \xi \in \Omega \backslash \eta\left(\varepsilon_{0}\right)
$$

Second order term in $\varepsilon$ :

$$
\begin{gathered}
\mu_{1}(\varepsilon z, s)=-\left[h_{2}^{\prime \prime}(z, s)-K^{2}(s) z m_{0}^{\prime}(z)-K(s) h_{1}^{\prime}(z, s)\right]+f^{\prime}\left(m_{0}(z)\right)\left[h_{2}(z, s)+\phi_{2}(\varepsilon z, s)\right] \\
-\varepsilon \Delta \phi_{1}(\varepsilon z, s)+f_{2}\left(m_{0}, m_{1}\right)(\varepsilon z, s)-G_{2,1}(\varepsilon z, s)
\end{gathered}
$$$$
\mu_{1}(\xi)=f^{\prime}(\bar{m}( \pm \infty)) \phi_{2}(\xi)+f_{2}\left(\bar{m}( \pm \infty), \phi_{1}(\xi)\right)-\varepsilon \Delta \phi_{1}(\xi)-G_{2,1}(\xi) \quad \text { for } \quad \xi \in \Omega \backslash \eta\left(\varepsilon_{0}\right) \text {. }
$$

More explicitly the $f_{2}$ term for $\xi \in \eta\left(\varepsilon_{0}\right)$ is given by

$$
f_{2}\left(m_{0}, m_{1}\right)(\varepsilon z, s)=\frac{1}{2} f^{\prime \prime}\left(m_{0}(z)\right)\left[h_{1}^{2}(z, s)+\phi_{1}^{2}(\varepsilon z, s)+2 \phi_{1}(\varepsilon z, s) h_{1}(z, s)\right],
$$

and by

$$
f_{2}\left(\bar{m}( \pm \infty), \phi_{1}(\xi)\right)=\frac{1}{2} f^{\prime \prime}(\bar{m}( \pm \infty)) \phi_{1}^{2}(\xi)
$$

for $\xi \in \Omega \backslash \eta\left(\varepsilon_{0}\right)$. 
$n$-th order term in $\varepsilon(3 \leqslant n \leqslant N)$ :

$$
\begin{aligned}
& \mu_{n-1}(\varepsilon z, s)=- h_{n}^{\prime \prime}(z, s)+a_{n}(z, s) m_{0}^{\prime}(z)+\sum_{i=1}^{n-1} a_{n-i}(z, s) h_{i}^{\prime}(z, s) \\
&+\sum_{i=1}^{n-2} b_{n-i}(z, s) \frac{d^{2}}{d s^{2}} h_{i}(z, s)+\mathbb{I}_{\{n \geqslant 4\}} \sum_{i=1}^{n-3} c_{n-i}(z, s) \frac{d}{d s} h_{i}(z, s) \\
& \quad-\varepsilon \Delta \phi_{n-1}(\varepsilon z, s)+f^{\prime}\left(m_{0}\right)\left[h_{n}(z, s)+\phi_{n}(\varepsilon z, s)\right] \\
& \quad+f_{n}\left(m_{0}, m_{1}, m_{2}, \ldots, m_{n-1}\right)(\varepsilon z, s)-G_{2, n-1}(\varepsilon z, s), \quad \xi \in \eta\left(\varepsilon_{0}\right),
\end{aligned}
$$

$$
\mu_{n-1}(\xi)=-\varepsilon \Delta \phi_{n-1}(\xi)+f^{\prime}( \pm 1) \phi_{n}(\xi)+f_{n}\left( \pm 1, \phi_{1}, \phi_{2}, \ldots, \phi_{n-1}\right)(\xi)-G_{2, n-1}(\xi),
$$

for $\xi \in \Omega \backslash \eta\left(\varepsilon_{0}\right)$.

Remainder term:

The remainder $\tilde{R}_{2}(\xi, t, \varepsilon) \equiv \tilde{R}_{2}$ is the following:

$$
\varepsilon \tilde{R}_{2}=\varepsilon^{N+1} G_{2, N}+\varepsilon^{N+1} A+\varepsilon^{N+2} \Delta \phi_{N}+E_{1}+\varepsilon^{N+1} B_{N+1} .
$$

From (4.5), (4.8) and (4.10) we obtain

$$
\sup _{(\xi, t) \in \Omega \times[0, T]}\left|\tilde{R}_{2}(\xi, t, \varepsilon)\right| \leqslant C \varepsilon^{N} .
$$

REMARK 4.2 Since, for $i=0, \cdots, N$, we required $\partial_{\nu} G_{2, i}=0$ on $\partial \Omega$ then the $\mu_{i}$ constructed in Theorem 3.1 satisfy $\partial_{\nu} \mu_{i}=0$ on $\partial \Omega$; to obtain $\partial_{\nu} m_{i}=0$ on $\partial \Omega$ it is enough to have $\partial_{\nu} \phi_{i}=0$ on $\partial \Omega$.

\subsection{Analysis of compatibility conditions}

Our aim is to analyze the equations obtained so far in the previous section. The strategy is to find first at each order in $\varepsilon$ the slowly varying part $\phi_{i}$ by solving the equations for $\xi \in \Omega \backslash \eta\left(\varepsilon_{0}, \Gamma_{t}^{(N)}\right)$. Then, we extend $\phi_{i}$ globally in $\Omega$ and determine the rapidly decaying part $h_{i}$ by solving the equations in $\xi \in \eta\left(\frac{\varepsilon_{0}}{2}, \Gamma_{t}^{(N)}\right)$. However here, in order to continue to arbitrary order, it is convenient to modify the way we extract the compatibility condition required for solving the equation for $h_{i}$. The modification is to add and subtract to each order a term of lower order $\varepsilon^{i+1} \alpha_{i}(s, \Gamma) \bar{m}^{\prime}(z)$, with $\alpha_{i}(\cdot, \Gamma) \in C^{\infty}(\Gamma)$. Adding and subtracting terms does not change, of course, the total quantity but it modifies the equation we obtain at each single order. In the following, for the sake of short notation, we write $\alpha_{i}(s) \equiv \alpha_{i}(s, \Gamma)$.

At each order $i \geqslant 1$ in $\varepsilon$ the associate function $m_{i}$ splits into two parts. The first is the function $\phi_{i}$ defined globally in $\Omega$ and satisfying Neumann condition on the boundary of $\Omega$ while the other part is the function $h_{i}$ which differs from zero only in a tubular neighborhood of $\Gamma, \eta\left(\varepsilon_{0}, \Gamma\right)$, and is exponentially decaying to zero far from $\Gamma$.

The zero order term is different in the sense that $m_{0}$ far from the interfaces relaxes exponentially fast to \pm 1 . We first state the following lemma, which is taken from [1]. We shall use this lemma to 
determine the condition for solvability of equations of the type (4.23), where $\mathcal{L}$ is the operator on $L^{2}(\mathbb{R})$ defined by

$$
\mathcal{L} g(z)=-g^{\prime \prime}(z)+f^{\prime}(\bar{m}(z)) g(z) .
$$

The operator $\mathcal{L}$ is self adjoint on $L^{2}(\mathbb{R})$ and has a null space spanned by $\bar{m}^{\prime}$. Therefore, the condition for solvability of $\mathcal{L} h_{1}=g$ is

$$
\int_{\mathbb{R}} g(z) \bar{m}^{\prime}(z) \mathrm{d} z=0 .
$$

Lemma 4.3 (see [1]) Let $A(z, s, t)$ defined for $z \in \mathbb{R}, s \in \Gamma, t \in[0, T]$. Assume that there exists $A^{ \pm}(s, t)$ such that $A(z, s, t)-A^{ \pm}(s, t)=\mathcal{O}\left(e^{-\alpha|z|}\right)$ as $|z| \rightarrow \infty$ for $s \in \Gamma$ and $t \in[0, T]$. Then for each $s \in \Gamma$ and $t \in[0, T]$

$$
\begin{aligned}
& (\mathcal{L} w)(z, s, t)=A(z, s, t) \quad \text { for } \quad z \in \mathbb{R}, \\
& w(0, s, t)=0, \quad w(\cdot, s, t) \in L^{\infty}(\mathbb{R}),
\end{aligned}
$$

has a solution if and only if

$$
\int_{\mathbb{R}} A(z, s, t) \bar{m}^{\prime}(z) d z=0 \quad \text { for all } \quad s \in \Gamma, \quad t \in[0, T] .
$$

In addition if the solution exists, then it is unique and satisfies

$$
D_{z}^{\ell}\left[w(z, s, t)+\frac{A^{ \pm}(s, t)}{f^{\prime}(1)}\right]=\mathcal{O}\left(e^{-\alpha|z|}\right) \quad \text { as } \quad|z| \rightarrow \infty, \quad \text { for } \quad \ell=0,1,2 .
$$

Furthermore, if $A(z, s, t)$ satisfies

$$
D_{s}^{m} D_{t}^{n} D_{z}^{\ell}\left[A(z, s, t)-A^{ \pm}(s, t)\right]=\mathcal{O}\left(e^{-\alpha|z|}\right),
$$

then

$$
D_{s}^{m} D_{t}^{n} D_{z}^{\ell}\left[w(z, s, t)+\frac{A^{ \pm}(s, t)}{f^{\prime}(1)}\right]=\circlearrowleft\left(e^{-\alpha|z|}\right)
$$

for all $m=0,1, \cdots, M, n=0,1, \cdots, N$, and $\ell=0,1, \cdots, L+2$. Further, since $\mathcal{L}$ is a preserving parity operator, the solution $w(z, s, t)$ is odd (even) with respect to $z$ if $A(z, s, t)$ is odd (even) with respect to $z$ for $s \in \Gamma$ and $t \in[0, T]$.

Remark 4.4 Note that if $A(\cdot, \cdot, \cdot) \in C^{\infty}(\mathbb{R} \times \Gamma \times[0, T])$ then the solution $w(\cdot, \cdot, \cdot)$ of the problem (4.23) is a function in $C^{\infty}(\mathbb{R} \times \Gamma \times[0, T])$. This would be always the case whenever we apply Lemma 4.3 .

The compatibility conditions must hold for every $\Gamma$ in $m$, and so, in our derivation we refer to $\Gamma:=\Gamma_{t}$.

4.2.1 Zero order term in $\varepsilon$. For $\xi \in \eta\left(\frac{\varepsilon_{0}}{2}\right)$ using (4.11) we obtain

$$
0=-\bar{m}^{\prime \prime}(z)+f(\bar{m}(z)) \quad \text { for } \quad z \in\left[-\frac{\varepsilon_{0}}{2 \varepsilon}, \frac{\varepsilon_{0}}{2 \varepsilon}\right],
$$

while (4.12) yields

$$
0=f( \pm 1) \quad \text { for } \quad \xi \in \Omega \backslash \eta\left(\varepsilon_{0}\right) .
$$

The above relations determine $m_{0}$, more specifically $m_{0}(z)=\bar{m}(z)$, where $\bar{m}$ solves the equation

$$
-m^{\prime \prime}(z)+f(m(z))=0, \quad z \in \mathbb{R} .
$$


4.2.2 First order term in $\varepsilon$ and determination of $V_{0}$ via the Fredholm alternative. As explained at the beginning of this section, it is convenient for solving (4.13) to add a term $\varepsilon \alpha_{1}(s, \Gamma) \bar{m}^{\prime}(z)$, $s \in \Gamma$ and $z \in \mathbb{R}$, with $\alpha_{1}(\cdot, \Gamma)$ to be determined. This term will be subtracted to the second order. Recalling the definition of $\mathcal{L}$, see (4.21), and adding $\varepsilon \alpha_{1}(s) \bar{m}^{\prime}(z)$, we write (4.13) as

$$
\mu_{0}(\varepsilon z, s)-f^{\prime}(\bar{m}(z)) \phi_{1}(\varepsilon z, s)-K(s) \bar{m}^{\prime}(z)+\varepsilon \alpha_{1}(s) \bar{m}^{\prime}(z)+G_{2,0}(\varepsilon z, s)=\left(\mathcal{L} h_{1}\right)(z, s),
$$

for $s \in \Gamma,|z| \leqslant \frac{\varepsilon_{0}}{2 \varepsilon}$. By (4.14) we obtain

$$
\phi_{1}(\xi)=\frac{\mu_{0}(\xi)+G_{2,0}(\xi)}{f^{\prime}(\bar{m}( \pm \infty))}, \quad \xi \in \Omega \backslash n\left(\varepsilon_{0}\right) .
$$

We extend this definition of $\phi_{1}$ globally in $\Omega$. We then insert (4.29) into (4.28) obtaining for $s \in \Gamma$, $|z| \leqslant \frac{\varepsilon_{0}}{2 \varepsilon}$

$$
\begin{aligned}
\mu_{0}(\varepsilon z, s)\left[1-\frac{f^{\prime}(\bar{m}(z))}{f^{\prime}( \pm 1)}\right]+\left(1-\frac{f^{\prime}(\bar{m}(z))}{f^{\prime}( \pm 1)}\right) G_{2,0}(\varepsilon z, s)-K(s) \bar{m}^{\prime}(z) & +\varepsilon \alpha_{1}(s) \bar{m}^{\prime}(z) \\
= & \left(\mathcal{L} h_{1}\right)(z, s) .
\end{aligned}
$$

Since the left hand side of (4.30) tends exponentially to 0 as $z \rightarrow \pm \infty$, if the solution of (4.30) exists (cf. Lemma 4.3), then it decays exponentially fast to 0 . We can, therefore, extend (4.30) for any $z$ in $\mathbb{R}$.

We prove the next result.

LEMMA 4.5 Set

$$
V_{0}^{(0)}(\xi, \Gamma)=\mathcal{J}_{\Gamma}\left[S\left\{K(\cdot)-\frac{\int_{\Gamma} K(\xi) \mathrm{d} S_{\xi}}{|\Gamma|}\right\}+\frac{1}{4}\left\{B_{0,0,0}(\cdot)-\frac{\int_{\Gamma} B_{0,0,0}(\xi) \mathrm{d} S_{\xi}}{|\Gamma|}\right\}\right](\xi), \quad \xi \in \Gamma,
$$

where

$$
S=\frac{1}{4} \int_{\mathbb{R}}\left(\bar{m}^{\prime}(z)\right)^{2} d z,
$$

and $B_{0,0,0}(\cdot)$ is defined in (4.39) while $\widetilde{J}_{\Gamma}$ is the Dirichlet-Neuman operator given by (A.1.4). Then there exists a uniquely determined $\alpha_{1}(\cdot, \Gamma) \in C^{\infty}(\Gamma)$ and a unique solution $h_{1}(\cdot, s)$ of (4.30) with $s \in \Gamma$, such that $h_{1}(0, s)=0$ and $h_{1}(\cdot, s) \in L^{\infty}(\mathbb{R})$. Moreover, $h_{1}(\cdot, s)$ for $s \in \Gamma$ and its derivatives with respect to $z$ decay exponentially fast to 0 as $z$ tends to $\pm \infty$. Further $h_{1}(\cdot, s)=$ $\tilde{h}_{1}(\cdot, s)+\varepsilon q_{\varepsilon}(\cdot, s)$ where $\tilde{h}_{1}(\cdot, s)$ is an even function of $z, \tilde{h}_{1}(0, s)=0$ and $\tilde{h}_{1}(\cdot, s) \in L^{\infty}(\mathbb{R})$.

Proof. We start determining $\tilde{h}_{1}$ as solution of

$$
\begin{aligned}
\mu_{0}(0, s)\left[1-\frac{f^{\prime}(\bar{m}(z))}{f^{\prime}( \pm 1)}\right]+\left(1-\frac{f^{\prime}(\bar{m}(z))}{f^{\prime}( \pm 1)}\right) G_{2,0}(0, s)-K(s) \bar{m}^{\prime}(z) & +\varepsilon \tilde{\alpha}_{1}(s) \bar{m}^{\prime}(z) \\
& =\left(\mathcal{L} \tilde{h}_{1}\right)(z, s) .
\end{aligned}
$$

Equation (4.33) differs from (4.30) only for terms of order $\varepsilon$. Namely $\left|G_{2,0}(0, s)-G_{2,0}(\varepsilon z, s)\right| \leqslant$ $C \varepsilon|z|$ and $\left|\mu_{0}(0, s)-\mu_{0}(\varepsilon z, s)\right| \leqslant C \varepsilon|z|$. For any fixed $s \in \Gamma$, the condition for the existence of 
$\tilde{h}_{1}$ requires that

$$
\begin{aligned}
\int_{\mathbb{R}} \mu_{0}(0, s)\left[1-\frac{f^{\prime}(\bar{m}(z))}{f^{\prime}( \pm 1)}\right] \bar{m}^{\prime}(z) d z & +\int_{\mathbb{R}} G_{2,0}(0, s)\left[1-\frac{f^{\prime}(\bar{m}(z))}{f^{\prime}( \pm 1)}\right] \bar{m}^{\prime}(z) d z \\
& =\left[K(s)-\varepsilon \tilde{\alpha}_{1}(s)\right] \int_{\mathbb{R}}\left(\bar{m}^{\prime}(z)\right)^{2} d z \quad \text { for } \quad s \in \Gamma .
\end{aligned}
$$

Taking into account that $\int_{\mathbb{R}} f^{\prime}(\bar{m}(z)) \bar{m}^{\prime}(z) d z=f(1)-f(-1)=0$ and $\int \bar{m}^{\prime}(z) d z=2$ formula (4.34) can be written as

$$
2\left[\mu_{0}(0, s)+G_{2,0}(0, s)\right]=\left[K(s)-\varepsilon \tilde{\alpha}_{1}(s)\right] \int_{\mathbb{R}}\left(\bar{m}^{\prime}(z)\right)^{2} d z \quad \text { for } \quad s \in \Gamma .
$$

Recalling (3.18) we obtain

$$
2 \mu_{0,0}(0, s)=-2 \tilde{\mu}_{0}(0, s)-2 G_{2,0}(0, s)+\left[K(s)-\varepsilon \tilde{\alpha}_{1}(s)\right] \int_{\mathbb{R}}\left(\bar{m}^{\prime}(z)\right)^{2} d z \quad \text { for } \quad s \in \Gamma .
$$

Further, we set

$$
\begin{gathered}
\mu_{0,0,0}(\xi)=2 \int_{\Gamma} V_{0}^{(0)}(\eta) G(\xi, \eta) \mathrm{d} S_{\eta}+c_{0}(t), \quad \xi \in \Omega, \\
\tilde{\mu}_{0,0,0}(\xi)=2\left\langle V_{0}\right\rangle_{\Gamma} \int_{\Gamma} G(\xi, \eta) \mathrm{d} S_{\eta}-\int_{\Omega} G(\xi, \eta) G_{1,0}(\eta) \mathrm{d} \eta, \quad \xi \in \Omega,
\end{gathered}
$$

and

$$
B_{0,0,0}(s)=-2\left[\tilde{\mu}_{0,0,0}(0, s)+G_{2,0}(0, s)\right] .
$$

It is immediate to see that

$$
\begin{gathered}
\mu_{0,0}(\xi)-\mu_{0,0,0}(\xi) \simeq \varepsilon \\
\tilde{\mu}_{0}(\xi)-\tilde{\mu}_{0,0,0}(\xi) \simeq \varepsilon .
\end{gathered}
$$

We first choose $V_{0}^{(0)}$ imposing for $s \in \Gamma$, cf. (4.36), the next identity

$$
2 \mu_{0,0,0}(\xi)=B_{0,0,0}(\xi)+K(\xi) \int_{\mathbb{R}}\left(\bar{m}^{\prime}(z)\right)^{2} d z \quad \xi \in \Gamma .
$$

Inserting (4.37) in (4.40) and integrating over $\Gamma$ we obtain that

$$
4 \int_{\Gamma} \mathrm{d} S_{\xi} \int_{\Gamma} V_{0}^{(0)}(\eta) G(\xi, \eta) \mathrm{d} S_{\eta}+2 c_{0}(t)|\Gamma|=\int_{\Gamma} K(\xi) \mathrm{d} S_{\xi} \int_{\mathbb{R}}\left(\bar{m}^{\prime}(z)\right)^{2} d z+\int_{\Gamma} B_{0,0,0}(\xi) \mathrm{d} S_{\xi},
$$

and therefore,

$$
\begin{aligned}
c_{0}(t)=\frac{1}{2|\Gamma|}\left[\int_{\Gamma} K(\xi) \mathrm{d} S_{\xi} \int_{\mathbb{R}}\left(\bar{m}^{\prime}(z)\right)^{2} d z+\int_{\Gamma}\right. & B_{0,0,0}(\xi) \mathrm{d} S_{\xi} \\
& \left.-4 \int_{\Gamma} \mathrm{d} S_{\xi} \int_{\Gamma} V_{0}^{(0)}(\eta) G(\xi, \eta) \mathrm{d} S_{\eta}\right] .
\end{aligned}
$$


We note that $c_{0}(t)$ is written in terms of the velocity field $V_{0}^{(0)}$ which still needs to be determined. We insert $c_{0}(t)$, as in (4.42), into (4.40) to obtain the equation determining $V_{0}^{(0)}$

$$
S_{\Gamma} V_{0}^{(0)}(\eta, \Gamma)=S\left[K(\eta)-\frac{\int_{\Gamma} K(\xi) \mathrm{d} S_{\xi}}{|\Gamma|}\right]+\frac{1}{4}\left[B_{0,0,0}(\xi)-\frac{\int_{\Gamma} B_{0,0,0}(\xi) \mathrm{d} S_{\xi}}{|\Gamma|}\right], \quad \eta \in \Gamma .
$$

Here, $S_{\Gamma}$ is the linear operator defined in (A.1.5) and $S$ the quantity defined in (4.32). Applying the Dirichlet-Neumann operator, see (A.1.6), we arrive at (4.31). The above determines first $V_{0}^{(0)}$ and then $c_{0}(t)$, see (4.42). Now since $V_{0}^{(0)}$ and $c_{0}(t)$ are chosen, we may then simply choose $\tilde{\alpha}_{1}(s)$ so that (4.34) is satisfied. Then for any $s \in \Gamma$, the existence of a unique $\tilde{h}_{1}$ satisfying $\tilde{h}_{1}(0, s)=0$ is assured; $\tilde{h}_{1}$ is exponentially decaying to zero as $|z| \rightarrow \infty$. Since the left-hand side of (4.33) is even, then the solution $\tilde{h}_{1}(\cdot, s)$ is even as a function of $z$.

Define $\varepsilon q_{\varepsilon}(\cdot, s)=h_{1}(\cdot, s)-\tilde{h}_{1}(\cdot, s)$ and subtract (4.33) to (4.30). We have that $q_{\varepsilon}(\cdot, s)$ satisfies

$$
\begin{array}{r}
\frac{1}{\varepsilon}\left[1-\frac{f^{\prime}(\bar{m}(z))}{f^{\prime}( \pm 1)}\right]\left[\mu_{0}(\varepsilon z, s)-\mu_{0}(0, s)+G_{2,0}(\varepsilon z, s)-G_{2,0}(0, s)\right]+[ \\
{\left[\alpha_{1}(s)-\tilde{\alpha}_{1}(s)\right] \bar{m}^{\prime}(z)} \\
=\left(\mathcal{L} q_{\varepsilon}\right)(z, s), \quad(4.43)
\end{array}
$$

where $\alpha_{1}(\cdot)$ must still be determined. We determine $\alpha_{1}(\cdot)$ so that the following solvability condition for $q_{\varepsilon}(\cdot, s)$ holds:

$$
\begin{aligned}
\frac{1}{\varepsilon} \int d z \bar{m}^{\prime}(z)\left[1-\frac{f^{\prime}(\bar{m}(z))}{f^{\prime}( \pm 1)}\right]\left[\mu_{0}(\varepsilon z, s)-\mu_{0}(0, s)+G_{2,0}(\varepsilon z, s)-G_{2,0}(0, s)\right] & \\
& +\left[\alpha_{1}(s)-\tilde{\alpha}_{1}(s)\right] \int d z\left(\bar{m}^{\prime}(z)\right)^{2}=0 .
\end{aligned}
$$

By Lemma 4.3 we have that for all $s \in \Gamma, q_{\varepsilon}(0, s)=0, q_{\varepsilon}(0, s) \in L^{\infty}(\mathbb{R})$.

REMARK 4.6 Let us denote by

$$
\mu_{0,0,0}^{F}=\mu_{0,0,0}+\tilde{\mu}_{0,0,0},
$$

the quantities defined in (4.37) and (4.38). Taking into account (2.19) and the definition of $\left\langle V_{0}\right\rangle_{\Gamma}=$ $\frac{1}{2\left|\Gamma_{t}\right|} \int_{\Omega} G_{1,0}(\eta) \mathrm{d} \eta$, see (3.17), we have that $\mu_{0,0,0}^{F}$ is the solution of

$$
\begin{gathered}
\Delta \mu(\xi)=-G_{1,0}(\xi) \quad \text { for } \quad \xi \in \Omega \backslash \Gamma, \\
\mu(\xi)=2 S K(\xi)-G_{2,0}(\xi) \quad \text { on } \quad \Gamma, \quad \partial_{\nu} \mu=0 \quad \text { on } \quad \partial \Omega .
\end{gathered}
$$

One deduces (4.46) by (4.40), taking into account the definition of $S$ and (4.39).

\subsubsection{Second order term in $\varepsilon$. From (4.16) we have that}

$$
\phi_{2}(\xi)=\frac{1}{f^{\prime}( \pm 1)}\left[\mu_{1}(\xi)-f_{2}\left(\bar{m}( \pm \infty), \phi_{1}(\xi)\right)+\varepsilon \Delta \phi_{1}+G_{2,1}(\xi)\right], \quad \xi \in \Omega \backslash \eta\left(\varepsilon_{0}\right) .
$$

As was done before, we extend the validity of (4.47) globally in $\Omega$. We insert (4.47) into (4.15). Further, we add and subtract to the next order $\varepsilon \alpha_{2}(s) \bar{m}^{\prime}(z)$ to obtain

$$
\mu_{1}(\varepsilon z, s)\left[1-\frac{f^{\prime}(\bar{m}(z))}{f^{\prime}( \pm 1)}\right]-A_{2}(z, s)+\varepsilon \alpha_{2}(s) \bar{m}^{\prime}(z)=\left(\mathcal{L} h_{2}\right)(z, s),
$$


where we set

$$
\begin{aligned}
A_{2}(z, s)=\left[G_{2,1}(\varepsilon z, s)+\varepsilon \Delta \phi_{1}(\varepsilon z, s)\right. & \left.-f_{2}\left( \pm 1, \phi_{1}\right)(\varepsilon z, s)\right]\left[1-\frac{f^{\prime}(\bar{m}(z))}{f^{\prime}( \pm 1)}\right] \\
& -K^{2}(s) z \bar{m}^{\prime}(z)-K(s) h_{1}^{\prime}(z, s)+\alpha_{1}(s) \bar{m}^{\prime}(z) .
\end{aligned}
$$

All the quantities in (4.49) have been already determined. Furthermore,

$$
\lim _{|z| \rightarrow \infty} A_{2}(z, s)=0, \quad s \in \Gamma
$$

the convergence being exponentially fast. As done before, we extend (4.48) in $\mathbb{R}$.

LEMMA 4.7 Set

$$
V_{1}^{(0)}(\xi, \Gamma)=\mathcal{J}_{\Gamma}\left[\frac{1}{4} B_{1}(\cdot)-\frac{1}{4|\Gamma|} \int_{\Gamma} B_{1}(s) \mathrm{d} s\right](\xi), \quad \xi \in \Gamma,
$$

where $B_{1}(s)$ is defined in (4.54) and $\mathcal{J}_{\Gamma}$ is the Dirichlet-Neumann operator. Then there exist uniquely determined $\alpha_{2}(\cdot, \Gamma) \in C^{\infty}(\Gamma)$ and $h_{2}(\cdot, s) \in t^{\infty}(\mathbb{R})$ with $h_{2}(0, s)=0$ for $s \in \Gamma$, solutions of (4.48). Moreover $h_{2}(\cdot, s)$ and its derivatives with respect to $z$ decay exponentially to 0 , as $z$ tends to $\pm \infty$.

Proof. The solvability condition, see (4.24), is satisfied provided that for $s \in \Gamma$ and $t \in[0, T]$ the next relation holds true

$$
\int_{\mathbb{R}} \mu_{1}(\varepsilon z, s)\left[1-\frac{f^{\prime}(\bar{m}(z))}{f^{\prime}(1)}\right] \bar{m}^{\prime}(z) d z=\int_{\mathbb{R}} A_{2}(z, s) \bar{m}^{\prime}(z) d z-\varepsilon \alpha_{2}(s) \int_{\mathbb{R}}\left(\bar{m}^{\prime}(z)\right)^{2} d z,
$$

where $\mu_{1}$ is defined in (3.34). The term $\tilde{\mu}_{1}$ of $\mu_{1}$ has been already completely determined. As in the previous case, still to be determined are the constant $c_{1}(t)$, the velocity $V_{1}^{(0)}$ and $\alpha_{2}(s)$. First, we write (4.52) as

$$
\int_{\mathbb{R}} \mu_{1,0}(\varepsilon z, s)\left[1-\frac{f^{\prime}(\bar{m}(z))}{f^{\prime}(1)}\right] \bar{m}^{\prime}(z) d z=B_{1}(s)-\varepsilon \alpha_{2}(s) \int_{\mathbb{R}}\left(\bar{m}^{\prime}(z)\right)^{2} d z,
$$

where

$$
B_{1}(s)=\int_{\mathbb{R}}\left\{A_{2}(z, s)-\tilde{\mu}_{1}(\varepsilon z, s)\left[1-\frac{f^{\prime}(\bar{m}(z))}{f^{\prime}(1)}\right]\right\} \bar{m}^{\prime}(z) d z .
$$

Let now

$$
\mu_{1,0,0}(\xi):=2 \int_{\Gamma} V_{1}^{(0)}(\eta) G(\xi, \eta) \mathrm{d} S_{\eta}+c_{1}(t), \quad \xi \in \Omega .
$$

Since $\mu_{1,0,0}(\xi)-\mu_{1,0}(\xi) \simeq \varepsilon$, we choose $V_{1}$ by imposing

$$
\int_{\mathbb{R}} \mu_{1,0,0}(0, s)\left[1-\frac{f^{\prime}(\bar{m}(z))}{f^{\prime}(1)}\right] \bar{m}^{\prime}(z) d z=B_{1}(s),
$$


and obtain

$$
\mu_{1,0,0}(0, s)=\frac{1}{2} B_{1}(s), \quad s \in \Gamma .
$$

Inserting (4.55) in (4.57) and integrating over $\Gamma$ we arrive at

$$
c_{1}(t)=\frac{1}{\left|\Gamma_{t}\right|}\left[\frac{1}{2} \int_{\Gamma_{t}} B_{1}(\eta) \mathrm{d} S_{\eta}-2 \int_{\Gamma_{t}} \mathrm{~d} S_{\xi} \int_{\Gamma_{t}} V_{1}^{(0)}(\eta) G(\xi, \eta) \mathrm{d} S_{\eta}\right] .
$$

Observe that $\int_{\Gamma} V_{1}^{(0)}(s) d s=0$ and let $S_{\Gamma}$ be the linear operator defined in (A.1.5). Then obviously, (4.57) can be written as

$$
S_{\Gamma} V_{1}^{(0)}(\xi)=\frac{1}{4} B_{1}(\xi)-\frac{1}{4|\Gamma|} \int_{\Gamma} B_{1}(s) d s, \quad \xi \in \Gamma,
$$

and thus, applying the Dirichlet-Neumann operator (see (A.1.6)) we obtain (4.51). This determines the (constant in $\xi) c_{1}(t)$. Now, since $V_{1}^{(0)}$ and $c_{1}(t)$ are determined we may choose $\alpha_{2}(s)$ so that (4.53) is satisfied.

REMARK 4.8 Notice that $\mu_{1,0,0}$ solves

$$
\begin{aligned}
& \Delta \mu_{1,0,0}=0 \quad \text { for } \quad \xi \in \Omega \backslash \Gamma, \\
& \mu_{1,0,0}(\xi)=\frac{1}{2} B_{1}(\xi) \quad \text { on } \quad \Gamma .
\end{aligned}
$$

4.2.4 $n$-th order term in $\varepsilon, 3 \leqslant n \leqslant N$. As previously, we determine the function $\phi_{n}$ for $\xi \in \Omega \backslash \eta\left(\varepsilon_{0}\right)$ from (4.18). Then, we extend the validity in $\Omega$ obtaining

$$
\phi_{n}(\xi)=\frac{1}{f^{\prime}(1)}\left[\mu_{n-1}(\xi)+\varepsilon \Delta \phi_{n-1}(\xi)-f_{n}\left( \pm 1, \phi_{1}, \phi_{2}, \cdots, \phi_{n-1}\right)(\xi)+G_{2, n-1}(\xi)\right], \quad \xi \in \Omega .
$$

We then insert (4.60) into (4.17), we add and subtract to the next order the quantity $\varepsilon \alpha_{n}(s) \bar{m}^{\prime}(z)$, to the left hand side of (4.17) and we obtain

$$
\mu_{n-1}(\varepsilon z, s)\left[1-\frac{f^{\prime}(\bar{m}(z))}{f^{\prime}(1)}\right]-A_{n}(z, s)+\varepsilon \alpha_{n}(s) \bar{m}^{\prime}(z)=\left(\mathcal{L} h_{n}\right)(z, s),
$$

where we set

$$
\begin{aligned}
A_{n}(z, s)= & -a_{n-1}(z, s) \bar{m}^{\prime}-\sum_{i=1}^{n-1}\left[a_{n-i}(z, s) h_{i}^{\prime}(z, s)\right]-\sum_{i=1}^{n-2} b_{n-i}(z, s) \frac{d^{2}}{d s^{2}} h_{i}(z, s) \\
& -\mathbb{I}_{n \geqslant 4} \sum_{i=1}^{n-3}\left[c_{n-i}(z, s) \frac{d}{d s} h_{i}(z, s)\right]-\varepsilon \Delta \phi_{n-1}(\varepsilon z, s)\left[1-\frac{f^{\prime}(\bar{m}(z))}{f^{\prime}(1)}\right] \\
+ & G_{2, n-1}(\varepsilon \xi)\left[1-\frac{f^{\prime}(\bar{m}(z))}{f^{\prime}(1)}\right]+\frac{f^{\prime}(\bar{m}(z))}{f^{\prime}( \pm 1)} f_{n}\left( \pm 1, \phi_{1}, \phi_{2}, \cdots, \phi_{n-1}\right)(\varepsilon z, s) \\
& -f_{n}\left(m_{0}, m_{1}, m_{2}, \cdots, m_{n-1}\right)(\varepsilon z, s) .
\end{aligned}
$$


It is easy to verify that for all $s \in \Gamma$

$$
\lim _{|z| \rightarrow \infty} A_{n}(z, s)=0
$$

where the convergence is exponentially fast. Namely there is no problem for those terms involving $\bar{m}^{\prime}, h_{i}(\cdot, s)$ and their derivatives because of the exponential convergence to zero of all these terms for all $s \in \Gamma$. For the remaining terms recall that $\lim _{|z| \rightarrow \infty} f^{\prime}(\bar{m}(z))=f^{\prime}( \pm 1), m_{i}=h_{i}+\phi_{i}$ with $h_{i}(z, s) \rightarrow 0$ as $|z| \rightarrow \infty$ for all $s \in \Gamma$, all limits being exponentially fast. Then one obtains immediately

$$
\lim _{|z| \rightarrow \infty}\left[1-\frac{f^{\prime}(\bar{m}(z))}{f^{\prime}( \pm 1)}\right] f_{n}\left( \pm 1, \phi_{1}, \phi_{2}, \cdots, \phi_{n-1}\right)(\varepsilon z, s)=0,
$$

exponentially fast also. We extend (4.61) to hold on all of $\mathbb{R}$ and regard it as an equation for $h_{n}(\cdot, s)$ for $s \in \Gamma$.

LEMmA 4.9 For any positive integer $n, n \leqslant N$, set

$$
V_{n-1}^{(0)}(\xi, \Gamma)=\mathcal{J}_{\Gamma} \frac{1}{4}\left[B_{n-1}(\cdot)-\frac{1}{|\Gamma|} \int_{\Gamma} B_{n-1}(s) d s\right] \text { for } \xi \in \Gamma,
$$

where $B_{n-1}(s)$ is defined in (4.66). Then there exist uniquely determined $\alpha_{n}(\cdot, \Gamma) \in C^{\infty}(\Gamma)$ and $h_{n}(\cdot, s) \in L^{\infty}(\mathbb{R})$ for $s \in \Gamma$ with $h_{n}(0, s)=0$, solutions of $(4.61)$. Moreover, $h_{n}(\cdot, s)$ for all $s \in \Gamma$, and its derivatives with respect to $z$ decay exponentially to 0 as $z \rightarrow \pm \infty$.

Proof. The solvability condition is satisfied provided that

$$
\int_{\mathbb{R}} \mu_{n-1}(\varepsilon z, s)\left[1-\frac{f^{\prime}(\bar{m}(z))}{f^{\prime}(1)}\right] \bar{m}^{\prime}(z) d z=\int_{\mathbb{R}} A_{n}(z, s) \bar{m}^{\prime}(z) d z-\varepsilon \alpha_{n}(s) \int_{\mathbb{R}}\left(\bar{m}^{\prime}(z)\right)^{2} d z .
$$

Since in view of (3.38) $\mu_{n-1}=\mu_{n-1,0}+\tilde{\mu}_{n-1}$ with $\tilde{\mu}_{n-1}$ being already determined to satisfy (4.64), we require that

$$
\int_{\mathbb{R}} \mu_{n-1,0}(\varepsilon z, s)\left[1-\frac{f^{\prime}(\bar{m}(z))}{f^{\prime}(1)}\right] \bar{m}^{\prime}(z) d z=B_{n-1}(s)-\varepsilon \alpha_{n}(s) \int_{\mathbb{R}}\left(\bar{m}^{\prime}(z)\right)^{2} d z,
$$

where

$$
B_{n-1}(s)=\int_{\mathbb{R}}\left\{A_{n}(z, s)-\tilde{\mu}_{n-1}(\varepsilon z, s)\left[1-\frac{f^{\prime}(\bar{m}(z))}{f^{\prime}(1)}\right]\right\} \bar{m}^{\prime}(z) d z .
$$

We set

$$
\mu_{n-1,0,0}(\xi)=2 \int_{\Gamma} V_{n-1}(\eta) G(\xi, \eta) \mathrm{d} S_{\eta}+c_{n-1}(t) .
$$

Since $\mu_{n-1,0}(\xi, t)-\mu_{n-1,0,0}(\xi, t) \simeq \varepsilon$ then we may determine $V_{n-1}$ by imposing

$$
\int_{\mathbb{R}} \mu_{n-1,0,0}(0, s)\left[1-\frac{f^{\prime}(\bar{m}(z))}{f^{\prime}(1)}\right] \bar{m}^{\prime}(z) d z=B_{n-1}(s),
$$


obtaining thus

$$
\mu_{n-1,0,0}(0, s)=\frac{1}{2} B_{n-1}(s) \quad s \in \Gamma .
$$

Inserting (4.67) in (4.69) and integrating over $\Gamma$ we get

$$
c_{n-1}(t)=\frac{1}{|\Gamma|}\left[\frac{1}{2} \int_{\Gamma} B_{n-1}(\eta) \mathrm{d} S_{\eta}-2 \int_{\Gamma} \mathrm{d} S_{\xi} \int_{\Gamma} V_{n-1}^{(0)}(\eta) G(\xi, \eta) \mathrm{d} S_{\eta}\right] .
$$

We insert (4.70) into (4.67), so, (4.69) gives

$$
S_{\Gamma} V_{n-1}^{(0)}(\xi)=\frac{1}{4}\left[B_{n-1}(\xi)-\frac{1}{|\Gamma|} \int_{\Gamma} B_{n-1}(s) \mathrm{d} s\right], \quad \xi \in \Gamma,
$$

and (4.63) follows. This determines $V_{n-1}^{(0)}$ first and then $c_{n-1}(t)$. Therefore, we may define $\alpha_{n}$ in order to satisfy (4.65).

\subsection{Proof of Theorem 4.1}

To complete the proof of Theorem 4.1 we need to estimate the remainder term (cf. (4.19)) given by

$$
\varepsilon R_{2}(\xi, t, \varepsilon)=\varepsilon \tilde{R}_{2}(\xi, t, \varepsilon)-\varepsilon^{N+1} \alpha_{N}(s(\xi), t) \bar{m}^{\prime}\left(\frac{d(\xi, \Gamma)}{\varepsilon}\right) .
$$

Since (4.20) holds true, we obtain that

$$
\sup _{\xi \in \Omega} \sup _{t \in[0, T]}\left|R_{2}(\xi, t, \varepsilon)\right| \leqslant C \varepsilon^{N} .
$$

So, Theorem 4.1 is proved.

\section{Proof of Theorem 2.5}

The proof of Theorem 2.5 is an immediate consequence of the following result.

Theorem 5.1 Take $N>1$ and $G_{1}$ and $G_{2}$ as in Assumption A1. There exist vector fields $V_{j}$, $j=0, \cdots,(N-1)$ and functions $m_{j}, j=0, \cdots, N$ from $m$ to $C^{\infty}(\Omega)$ as in the Ansatz 2.2 such that the following holds. For any $\Gamma_{0} \in m$, choose $k_{0} \geqslant k\left(\Gamma_{0}\right)$, set $\varepsilon_{0}=\frac{1}{2 k_{0}}$ and let $T$ be the lifetime of the solution of (2.7) in $m$, according to (2.3). Then for all $t<T$ and for all $\varepsilon \in\left(0, \varepsilon_{0}\right]$ we can construct $\left(\tilde{m}^{(N)}, \tilde{\mu}^{(N-1)}\right) \in C^{\infty}(\Omega \times[0, T])$ where $\tilde{m}^{(N)}$ is an $\varepsilon^{N}$ modification of $m^{(N)}$, i.e. $\sup _{(\xi, t) \in \Omega \times[0, T]}\left|\tilde{m}^{(N)}(\xi, t)-m^{(N)}(\xi, t)\right| \leqslant C \varepsilon^{N}$ and $\tilde{\mu}^{(N-1)}$ is an $\varepsilon^{N-1}$ modification of $\mu^{(N-1)}$, i.e. $\sup _{(\xi, t) \in \Omega \times[0, T]}\left|\tilde{\mu}^{(N-1)}(\xi, t)-\mu^{(N-1)}(\xi, t)\right| \leqslant C \varepsilon^{N-1}$ satisfying

$$
\begin{gathered}
\partial_{t} \tilde{m}^{(N)}(\xi, t)=\Delta \tilde{\mu}^{(N-1)}(\xi, t)+\sum_{j=0}^{N-1} \varepsilon^{j} G_{1, j}(\xi) \quad \text { in } \quad \Omega \times(0, T), \\
\tilde{\mu}^{(N-1)}(\xi, t)=-\varepsilon \Delta \tilde{m}^{(N)}(\xi, t)+\frac{1}{\varepsilon} f\left(\tilde{m}^{(N)}(\xi, t)\right)-\sum_{j=0}^{N-1} \varepsilon^{j} G_{2, j}(\xi)+R^{(N)}(\xi, t, \varepsilon) \\
\operatorname{in} \Omega \times(0, T),
\end{gathered}
$$

where

$$
\sup _{\xi \in \Omega} \sup _{t \in[0, T]}\left|R^{(N)}(\xi, t, \varepsilon)\right| \leqslant C \varepsilon^{N-1}
$$


Further, $\tilde{\mu}^{(N-1)}(\cdot, t)$ and $\tilde{m}^{(N)}(\cdot, t)$, for $t \in[0, T]$, satisfy Neumann homogeneous boundary conditions on the boundary of $\Omega$. In addition

$$
\sup _{t \in[0, T]} \sup _{\xi \in \Omega}\left|\tilde{\mu}^{(N-1)}(\xi, t)-\mu_{0,0,0}^{F}(\xi, t)\right| \leqslant C \varepsilon,
$$

where $\mu_{0,0,0}^{F}$ is the solution of

$$
\Delta \mu(\xi)=-G_{1,0}(\xi), \quad \xi \in \Omega \backslash \Gamma_{t}^{(N)},
$$

subject to the boundary conditions

$$
\mu(\xi)=2 S K(\xi)-G_{2,0}(\xi) \quad \text { on } \quad \Gamma_{t}^{(N)}, \quad \partial_{\nu} \mu=0 \quad \text { on } \quad \partial \Omega
$$

and

$$
\begin{gathered}
\sup _{t \in[0, T]} \sup _{\xi \in \eta\left(\varepsilon_{0}, \Gamma_{t}^{(N)}\right)}\left|\tilde{m}^{(N)}(\xi, t)-\bar{m}\left(\frac{d\left(\xi, \Gamma_{t}^{(N)}\right)}{\varepsilon}\right)\right| \leqslant C \varepsilon, \\
\sup _{t \in[0, T]} \sup _{\left.\xi \in \Omega \backslash \eta\left(\varepsilon_{0}\right), \Gamma_{t}^{(N)}\right)}\left|\tilde{m}^{(N)}(\xi, t) \mp 1\right| \leqslant C \varepsilon .
\end{gathered}
$$

Proof. Set

$$
\tilde{m}^{(N)}(\xi, t)=m^{(N)}(\xi, t)-\int_{0}^{t} \bar{R}_{1}(\tau, \varepsilon) d \tau
$$

where

$$
\bar{R}_{1}(t, \varepsilon)=\frac{1}{|\Omega|} \int_{\Omega} R_{1}(\xi, t, \varepsilon) \mathrm{d} \xi
$$

and $R_{1}(\xi, t, \varepsilon)$ is the remainder in Theorem 3.1, defined in (3.14) and estimated in (3.3) and (3.4). Let us denote by

$$
\tilde{\mu}^{(N-1)}(\xi, t)=\mu^{(N-1)}(\xi, t)+v(\xi, t)
$$

where $v(\xi, t)$ solves

$$
\begin{gathered}
\Delta v(\xi, t)=R_{1}(\xi, t, \varepsilon)-\bar{R}_{1}(t, \varepsilon) \text { for } \xi \in \Omega, \\
\partial_{\nu} v=0 \text { on } \partial \Omega,
\end{gathered}
$$

with the further requirement

$$
\int_{\Omega} v(\xi, t) \mathrm{d} \xi=0, \quad t \in[0, T] .
$$

Since $\left|R_{1}(\xi, t, \varepsilon)\right| \leqslant C(T) \varepsilon^{N-1}$ we have that $|v(\xi, t)| \leqslant C \varepsilon^{N-1}$. The functions $\tilde{m}^{(N)}$ and $\tilde{\mu}^{(N-1)}$ satisfy (5.1). Namely the first equation of (5.1) is satisfied by Theorem 3.1 and by construction, see (5.7) and (5.9). The second equation is obtained from Theorem 4.1 adding and subtracting terms to obtain $\tilde{\mu}^{(N-1)}$ and $\tilde{m}^{(N)}$. We obtain

$$
\tilde{\mu}^{(N-1)}=\mu^{(N-1)}+v=-\varepsilon \Delta \tilde{m}^{(N)}+\frac{1}{\varepsilon} f\left(\tilde{m}^{(N)}\right)+R^{(N)},
$$


where

$$
R^{(N)} \equiv R^{(N)}(\xi, t, \varepsilon)=\frac{1}{\varepsilon}\left[f\left(\tilde{m}^{(N)}+\int_{0}^{t} \bar{R}_{1}(\tau, \varepsilon) \mathrm{d} \tau\right)-f\left(\tilde{m}^{(N)}\right)\right]+R_{2}+v,
$$

and $R_{2}$ is the remainder in Theorem 4.1, see (4.71). Since $\bar{R}_{1}=\mathcal{O}\left(\varepsilon^{N}\right), R_{2}=\mathcal{O}\left(\varepsilon^{N}\right), v=$ $\mathcal{O}\left(\varepsilon^{N-1}\right)$ and

$$
\frac{1}{\varepsilon}\left[f\left(\tilde{m}^{(N)}+\int_{0}^{t} \bar{R}_{1}(\tau, \varepsilon) \mathrm{d} \tau\right)-f\left(\tilde{m}^{(N)}\right)\right] \leqslant \frac{C}{\varepsilon} \int_{0}^{t} \bar{R}_{1}(\tau, \varepsilon) \mathrm{d} \tau=\mathcal{O}\left(\varepsilon^{N-1}\right),
$$

then the second equation of (5.1) is satisfied as well. In Remark 4.6 it is explained that if $\mu_{0,0,0}^{F}=$ $\mu_{0,0,0}+\tilde{\mu}_{0,0,0}$, where $\mu_{0,0,0}$ and $\tilde{\mu}_{0,0,0}$ are the quantities defined in (4.37) and (4.38) then it verifies (5.3) and (5.4). The relation (5.2) is then immediate from their definition and Theorem 3.1. The (5.5) and (5.6) are satisfied by construction of the $m^{(N)}$. Theorem 2.5 is then proved.

\section{Appendix}

\section{A.1. The Dirichlet-Neumann operator}

We recall in this section the main properties of the Dirichlet-Neumann operator which we have been using through the paper. Some of these results were already presented in the Appendix of [14]. Let $G(\xi, \eta)$ be the Green's function in $\Omega$ defined in (2.19) and verifying (2.20). To define the DirichletNeumann operator we consider the following single layer potentials. Given a smooth function $h$ defined on $\Gamma \in m$, consider the single layer potential

$$
\phi_{h}(\xi)=\int_{\Gamma} G(\xi, \eta) h(\eta) \mathrm{d} S_{\eta},
$$

where $\mathrm{d} S_{\eta}$ denotes the arc length measure along $\Gamma$. The function $\phi_{h}$ satisfies a Neumann boundary condition on $\partial \Omega$, and also the equation

$$
\Delta \phi_{h}(\xi)=h(\xi)-\frac{1}{|\Omega|} \int_{\Gamma} h(\eta) \mathrm{d} S_{\eta}, \quad \xi \in \Gamma
$$

The curve $\Gamma$ separates $\Omega$ in two subsets. We denote by $\Omega_{\Gamma}^{-}$the interior of $\Gamma$ and by $\Omega_{\Gamma}^{+}$its exterior. We denote by $n$ the unit outer normal to $\Omega_{\Gamma}^{-}$. There is a discontinuity in the normal derivatives of $\phi_{h}$ across $\Gamma$, so, we have that

$$
h(\xi)=\frac{1}{2}\left[\partial_{\nu} \phi_{h}\right]_{\Gamma}(\xi),
$$

where the right-hand side denotes the jump of the normal derivatives at $\xi \in \Gamma$, i.e.,

$$
\left[\partial \nu \phi_{h}\right]_{\Gamma}(\xi)=\left(\partial_{\nu} \phi_{h}\right)_{\Omega_{\Gamma}^{+}}(\xi)-\left(\partial_{\nu} \phi_{h}\right)_{\Omega_{\Gamma}^{-}}(\xi) .
$$

This is a well known result from potential theory [24]. For $\xi$ away from $\Gamma$,

$$
\Delta \phi_{h}(\xi)=-\frac{1}{|\Omega|} \int_{\Gamma} h(\eta) \mathrm{d} S_{\eta}
$$


Thus, the single layer potential is harmonic away from $\Gamma$ if and only if $\int_{\Gamma} h(\xi) \mathrm{d} S_{\xi}=0$. Otherwise, it is subharmonic or superharmonic, according to whether $-\int_{\Gamma} h(\xi) \mathrm{d} S_{\xi}$ is positive or negative. Every continuous function $\phi$ harmonic away from $\Gamma$, satisfying the Neumann boundary condition and the following relation

$$
\int_{\Omega} \phi(\xi) \mathrm{d} \xi=0
$$

is the single layer potential of a uniquely determined function $h$ defined on $\Gamma$ and satisfying

$$
\int_{\Gamma} h(\xi) \mathrm{d} S_{\xi}=0
$$

Indeed, if $\phi_{h}$ is such a single layer potential, then from (2.20), we get that $\int_{\Omega} \phi_{h}(\xi) \mathrm{d} \xi=0$.

On the other hand, let $\phi$ be any continuous function that is harmonic on $\Omega_{\Gamma}^{-}$and $\Omega_{\Gamma}^{+}$, and which satisfies (A.1.2). Let us define $h$ in $\Gamma$ by

$$
h(\xi)=\frac{1}{2}\left[\partial_{\nu} \phi\right]_{\Gamma}(\xi),
$$

and refer to this as the Neumann data for $\phi$. By the divergence theorem we obtain

$$
2 \int_{\Gamma} h(\xi) \mathrm{d} S_{\xi}=\int_{\Gamma} \partial_{\nu} \phi^{+} \mathrm{d} S_{\xi}-\int_{\Gamma} \partial_{\nu} \phi^{-} \mathrm{d} S_{\xi}=-\int_{\Omega \backslash \Gamma} \Delta \phi \mathrm{d} \xi=0,
$$

where $\phi^{ \pm}$denotes the restriction of $\phi$ in $\Omega^{ \pm}$. Hence, $h$ satisfies (A.1.3).

Notice that $\phi-\phi_{h}$ satisfies the Neumann boundary conditions and

$$
\left[\partial_{\nu}\left(\phi-\phi_{h}\right)\right]_{\Gamma}(\xi)=0 .
$$

This means that $\phi-\phi_{h}$ is a constant. Since the integral is zero then $\phi=\phi_{h}$. This proves the one to one correspondence between single layer potentials of functions $h$ satisfying (A.1.3), and continuous functions $\phi$ that are harmonic on $\Omega_{\Gamma}^{-}$and $\Omega_{\Gamma}^{+}$, and satisfy (A.1.2).

Next, given a continuous function $\phi$ that is harmonic on $\Omega_{\Gamma}^{-}$and $\Omega_{\Gamma}^{+}$, whether or not (A.1.2) is satisfied, we define the function $g$ on $\Gamma$ by $g:=\left.\phi\right|_{\Gamma}$. We naturally refer to $g$ as the Dirichlet data for $\phi$. The Neumann data is $\left[\partial_{\nu} \phi\right]_{\Gamma}$. The Dirichlet-Neuman operator $\mathcal{J}_{\Gamma}$ is defined by

$$
\mathcal{J}_{\Gamma} g=\frac{1}{2}\left[\partial_{\nu} \phi\right]_{\Gamma},
$$

where $\phi$ is the continuous function that is harmonic in $\Omega_{\Gamma}^{-}$and $\Omega_{\Gamma}^{+}$, with $\left.\phi\right|_{\Gamma}=g$.

A simple argument shows that $\tau_{\Gamma}$ is a positive Hermitian operator. Indeed, let $\psi$ be continuous on $\Omega$, and harmonic on $\Omega_{\Gamma}^{-}$and $\Omega_{\Gamma}^{+}$, with $\left.\psi\right|_{\Gamma}=h$. Then it follows that

$$
\begin{aligned}
2 \int_{\Gamma} h \mathcal{J}_{\Gamma} g \mathrm{~d} s & =\int_{\Gamma} \psi\left[\partial_{\nu} \phi\right]_{\Gamma} \mathrm{d} s \\
& =-\int_{\Omega_{\Gamma}^{+}} \nabla \cdot(\psi(\nabla \phi)) \mathrm{d} \xi-\int_{\Omega_{\Gamma}^{-}} \nabla \cdot(\psi(\nabla \phi)) \mathrm{d} \xi \\
& =-\int_{\Omega} \nabla \psi \cdot \nabla \phi \mathrm{d} \xi .
\end{aligned}
$$


Taking $h=1$, so that $\psi=1$, we further see that the range of $\tau_{\Gamma}$ is orthogonal to the constants. We let $\tau_{\Gamma}$ denote the Friedrichs extension of $\tau_{\Gamma}$. It is easy to see, and well known, that the form domain of $\mathcal{J}_{\Gamma}$ is the Sobolev space $H^{1 / 2}(\Gamma)$, and that the kernel consists exactly of the constants. There is an explicit formula for the inverse of $\mathcal{J}_{\Gamma}$ restricted to the orthogonal complement of the constants; we denote this by $\delta_{\Gamma}$. Indeed, let $v$ be any function on $\Gamma$ with $\int_{\Gamma} v(s) \mathrm{d} s=0$. Since the single layer potential $\phi_{v}$ for $v$ has Neumann data $v$, all we need to do is to subtract a constant to make this function orthogonal to the constants on $\Gamma$, instead of being orthogonal to the constants on $\Omega$. Therefore, the inverse $\delta_{\Gamma}$ is given by

$$
\delta_{\Gamma} v(\xi)=\int_{\Gamma} G(\xi, \eta) v(\eta) \mathrm{d} S_{\eta}-\frac{1}{|\Gamma|} \int_{\Gamma} \int_{\Gamma} G(\xi, \eta) v(\eta) \mathrm{d} S_{\eta} \mathrm{d} S_{\xi}, \quad \xi \in \Gamma .
$$

It is easily checked that the inverse operator is self adjoint on the orthogonal complement of the constants. Now let $h$ be an arbitrary smooth function on $\Gamma$ satisfying $\int_{\Gamma} h(s) \mathrm{d} s=0$, and consider the single layer potential

$$
\phi(\xi)=\int_{\Gamma} G(\xi, \eta) h(\eta) \mathrm{d} S_{\eta}, \quad \xi \in \Omega .
$$

In general, the Dirichlet data for $\phi$ do not integrate to zero on $\Gamma$ and hence, are not directly related to the Neumann data through the Dirichlet-Neumann operator. However, we can correct this by subtracting a constant and defining the function

$$
\tilde{\phi}(\xi)=\phi(\xi)-\frac{1}{|\Gamma|} \int_{\Gamma} \phi(\eta) \mathrm{d} S_{\eta}
$$

Then obviously we have

$$
\begin{aligned}
& \left.\tilde{\phi}\right|_{\Gamma}=\delta_{\Gamma} h, \\
& h=\mathcal{J}_{\Gamma} \tilde{\phi} .
\end{aligned}
$$

We can now express the vector field $V$ driving the Mullins-Sekerka flow as

$$
V=\mathcal{J}_{\Gamma}\left(K-\frac{1}{|\Gamma|} \int_{\Gamma} K(s) \mathrm{d} s\right) .
$$

We close by establishing notation for the two harmonic extension operators that will arise throughout what follows:

The Neumann harmonic extension operator $\varepsilon_{\Gamma, N}$ is defined by

$$
\left(\varepsilon_{\Gamma, N} v\right)(\xi)=\int_{\Gamma} G(\xi, \eta) v(\eta) \mathrm{d} S_{\eta}-\frac{1}{|\Gamma|} \int_{\Gamma} \int_{\Gamma} G(\xi, \eta) v(\eta) \mathrm{d} S_{\eta} \mathrm{d} S_{\xi}, \quad \xi \in \Omega,
$$

where $v$ is a function on $\Gamma$ satisfying

$$
\int_{\Gamma} v(\xi) \mathrm{d} S_{\xi}=0
$$

Notice that $\left(\varepsilon_{\Gamma, N} v\right)(\xi)$ is the unique function that is continuous on $\Omega$, harmonic on $\Omega \backslash \Gamma$ satisfying Neumann boundary conditions on $\partial \Omega$, with Neumann data $v$, and with zero integral over $\Gamma$. 
The Dirichlet harmonic extension operator $\varepsilon_{\Gamma, D}$ is defined by setting $\varepsilon_{\Gamma, D} g(\xi)$ to be the harmonic function $\phi$ on $\Omega \backslash \Gamma$ with Neumann boundary conditions on $\partial \Gamma$, and $\left.\phi\right|_{\Gamma}=g$. Here, there is no restriction on the integral of $g$ over $\Gamma$. Naturally, the Dirichlet extension can be expressed in terms of the Neumann extension and the Dirichlet-Neumann operator. Relations (A.1.5) and (A.1.8) give that

$$
\varepsilon_{\Gamma, D} g(\xi)=\varepsilon_{\Gamma, N}\left(\Im_{\Gamma}\left(g-\frac{1}{|\Gamma|} \int_{\Gamma} g(\eta) \mathrm{d} S_{\eta}\right)\right)(\xi)+\frac{1}{|\Gamma|} \int_{\Gamma} g(\eta) \mathrm{d} S_{\eta}, \quad \xi \in \Omega
$$

\section{A.2. The expansion in $\varepsilon$ of the Laplacian in local coordinates}

Let $f(z, s)$, with $z=\frac{d}{\varepsilon}$, be a $C^{2}$ function from $\mathbb{R} \times \Gamma$ to $\mathbb{R}$. Then, in the two dimensional case, we have that

$$
\begin{aligned}
\varepsilon^{2} \Delta f(z, s) & =\frac{1}{1-K(s) \varepsilon z}\left\{\left((1-K(s) \varepsilon z) f_{z}\right)_{z}+\varepsilon^{2}\left(\frac{f_{s}}{1-K(s) \varepsilon z}\right)_{s}\right\} \\
& =f_{z z}-\varepsilon K(s) f_{z} \frac{1}{1-K(s) \varepsilon z}+\varepsilon^{2} \frac{f_{s s}}{(1-K(s) \varepsilon z)^{2}}+\varepsilon^{3} f_{s} \frac{\frac{d}{d s} K(s) z}{(1-K(s) \varepsilon z)^{3}}
\end{aligned}
$$

Recalling that for $|x|<1$ it holds that

$$
\frac{1}{(1-x)}=\sum_{n=0}^{\infty} x^{n}, \quad \frac{1}{(1-x)^{2}}=\sum_{n=0}^{\infty} n x^{n-1}, \quad \frac{1}{(1-x)^{3}}=\frac{1}{2} \sum_{n=0}^{\infty} n(n-1) x^{n-2},
$$

we may rewrite (A.2.1) as follows

$$
\varepsilon^{2} \Delta f=f_{z z}+\sum_{n=0}^{\infty} \varepsilon^{n+1}\left\{a_{n+1}(z, s) f_{z}+b_{n+1}(z, s) f_{s s}+c_{n+1}(z, s) f_{s}\right\},
$$

where

$$
\begin{aligned}
& a_{n+1}(z, s)=-K^{n+1}(s) z^{n}, \\
& b_{n+1}(z, s)=n K^{n-1}(s) z^{n-1}, \\
& c_{n+1}(z, s)=\frac{1}{2} n(n-1) z^{n-1} K^{n-2}(s) \frac{d}{d s} K(s) .
\end{aligned}
$$

\section{A.3. Proof of Theorem 2.7}

The proof goes very much as in [1, Theorem 2.1]. Hence we outline only those points where the presence of the $G_{1}$ and $G_{2}$ makes a difference. First of all the constructed functions $m^{(N)}(t)$, $t \in[0, T]$ satisfy the requirements needed to apply the spectral estimates proven by [2] and [15]. Namely, see (2.8), (2.10) and Lemma 4.5, we can always write for $\xi \in \Omega$ and $\in[0, T]$

$m^{(N)}(\xi, t)=m_{0}\left(\frac{d\left(\xi, \Gamma_{t}^{(N)}\right)}{\varepsilon}\right)+\varepsilon \tilde{h}_{1}\left(\frac{d\left(\xi, \Gamma_{t}^{(N)}\right)}{\varepsilon}, s\left(\xi, \Gamma_{t}^{(N)}\right)\right)+\varepsilon^{2} q^{\varepsilon}\left(\xi, \Gamma_{t}^{(N)}\right)+\varepsilon \phi^{\varepsilon}\left(\xi, \Gamma_{t}^{(N)}\right)$, 
where $m_{0}$ is given in (2.9), $\tilde{h}_{1}(\cdot, s)$ is the function determined in Lemma 4.5 which is even as function of $z \in \mathbb{R}$ for any $s \in \Gamma_{t}^{(N)}$, equal to 0 in $\Omega \backslash \eta\left(\varepsilon_{0}\right)$ and when $d\left(\xi, \Gamma_{t}^{(N)}\right)=0$. We denote by $\varepsilon^{2} q^{\varepsilon}\left(\xi, \Gamma_{t}^{(N)}\right)$ the remaining functions in the expansion of $m^{(N)}$ which are equal to zero in $\Omega \backslash \eta\left(\varepsilon_{0}\right)$ and by $\varepsilon \phi^{\varepsilon}\left(\xi, \Gamma_{t}^{(N)}\right)$ the corrections to \pm 1 in $\Omega \backslash \eta\left(\varepsilon_{0}\right)$. Recall $m^{(N)}(\cdot, t)$ are $C^{\infty}(\Omega)$ for any $t \in[0, T]$. We immediate have, since $\bar{m}(\cdot)$ is odd while $h_{1}(\cdot, s)$ is even,

$$
\int_{\mathbb{R}} \tilde{h}_{1}(z, s)\left(\bar{m}^{\prime}(z)\right)^{2} f^{\prime \prime}(\bar{m}(z)) d z=6 \int_{\mathbb{R}} \tilde{h}_{1}(z, s)\left(\bar{m}^{\prime}(z)\right)^{2} \bar{m}(z) d z=0, \quad \forall s \in \Gamma_{t}^{(N)} .
$$

This is one of the requirement needed to apply the spectral estimates. The remaining requirements are immediately satisfied by the smoothness of $m^{(N)}(\cdot, t)$ and by the fact that the $\Phi_{j}, j=1, \ldots, N$ in the expansion of $m^{(N)}$ satisfy a global Lipschitz bound independent on $\varepsilon$.

Then one proceeds as in [1]. Write (1.8) as the the following:

$$
\begin{aligned}
& \partial_{t} m^{\varepsilon}=\Delta \mu^{\varepsilon}+G_{1} \quad \text { in } \Omega_{T}, \\
& \mu^{\varepsilon}=-\varepsilon \Delta m^{\varepsilon}+\frac{1}{\varepsilon} f\left(m^{\varepsilon}\right)-G_{2} \quad \text { in } \Omega_{T}, \\
& m^{\varepsilon}(\xi, 0)=m_{0}^{\varepsilon}(\xi), \quad \xi \in \Omega, \\
& \partial_{\nu} m^{\varepsilon}=\partial_{\nu} \Delta m^{\varepsilon}=0 \quad \text { on } \partial \Omega,
\end{aligned}
$$

and (2.12) the the following:

$$
\begin{aligned}
& \partial_{t} m^{(N)}=\Delta \mu^{(N)}+\sum_{j=0}^{N-1} \varepsilon^{j} G_{1, j} \quad \text { in } \Omega_{T}, \\
& \mu^{(N)}=-\varepsilon \Delta m^{(N)}+\frac{1}{\varepsilon} f\left(m^{(N)}\right)-\sum_{j=0}^{N-1} \varepsilon^{j} G_{2, j}+R^{(N)} \text { in } \Omega_{T}, \\
& m^{(N)}(\xi, 0)=m_{0}^{\varepsilon}(\xi), \quad \xi \in \Omega, \\
& \partial_{\nu} m^{(N)}=\partial_{v} \Delta m^{(N)}=0 \quad \text { on } \partial \Omega .
\end{aligned}
$$

Define $R:=m^{\varepsilon}-m^{(N)}$. Then integrating $R$ in space, by (A.3.1) and (A.3.2) and the fact that $R(\xi, 0)=0$, we obtain

$$
\begin{aligned}
\int_{\Omega} R(\xi, t) d \xi & =\int_{\Omega}\left(m^{\varepsilon}-m^{(N)}\right) d \xi=\int_{\Omega} d \xi \int_{0}^{t} \partial_{s}\left(m^{\varepsilon}-m^{(N)}\right) d s \\
& =\int_{\Omega} d \xi \int_{0}^{t}\left(\Delta \mu^{\varepsilon}+G_{1}-\Delta \mu^{(N)}-\sum_{j=0}^{N-1} \varepsilon^{j} G_{1, j}\right) d s \\
& =\int_{0}^{t} d s \int_{\Omega} \Delta\left[\mu^{\varepsilon}-\mu^{(N)}\right] d \xi+\varepsilon^{N} \int_{0}^{t} d s \int_{\Omega}\left[G_{1, N}\right] d \xi d s \\
& =\int_{0}^{t} d s \int_{\Omega} \Delta\left[\mu^{\varepsilon}-\mu^{(N)}\right] d \xi=0,
\end{aligned}
$$

since $\partial_{\nu}\left[\mu^{\varepsilon}-\mu^{(N)}\right]=0$ on $\partial \Omega$, and (2.21) holds. Note that we need (and used for the above) $\partial_{\nu} G_{2}=\partial_{\nu} R^{(N)}=0$ on $\partial \Omega$ in order to have $\partial_{v}\left[\mu^{\varepsilon}-\mu^{(N)}\right]=0$ on $\partial \Omega$. In addition, we need the 
residual $R^{(N)}$ satisfying a Neumann condition, i.e.,

$$
\partial_{v} R^{(N)}=0 \quad \text { on } \partial \Omega
$$

which is true by construction, cf. system (5.1) and the b.c. on system solutions in the statement of Theorem 5.1.

Hence, since by (A.3.3), for any $t \in[0, T] \int_{\Omega} R(\xi, t) d \xi=0$ then there exists unique $\psi(\xi, t)$ such that

$$
\begin{aligned}
& -\Delta \psi(\cdot, t)=R(\cdot, t) \quad \text { in } \Omega, \\
& \partial_{\nu} \psi(\cdot, t)=0 \quad \text { on } \partial \Omega, \\
& \int_{\Omega} \psi(\cdot, t)=0 \quad \text { for any } t \in[0, T] .
\end{aligned}
$$

At this point one can continue the proof as in [1].

Acknowledgments. DA and GK are supported under the "ARISTEIA" Action of the "Operational programme education and lifelong learning" and is co-funded by the European Social Fund (ESF) and National Resources. EO has been supported by MIUR-PRIN 2011-2013.

\section{REFERENCES}

1. Alikakos, N. D., Bates, P. W. \& Chen, X, Convergence of the Cahn-Hilliard equation to the HeleShaw Model, Arch. Rat. Mech. Anal. 128 (1994), 165-205. Zb10828. 35105 MR1308851

2. Alikakos, N. D. \& Fusco, G., The spectrum of the Cahn-Hilliard operator for generic interfaces in higher space dimensions, Indiana University Math. J. 42 (1993), 637-674. Zbl0798. 35123 MR1237062

3. Antal, T., Droz, M., Magnin, J. \& RÁcz, Z., Formation of Liesengang patterns: A spinodal decomposition scenarion, Phys. Rev. Lett. 83 (1999), 2880-2883. Zbl1270 . 35040

4. Antonopoulou, D. C., Bates, P. W. \& Karali, G. D., Motion of a droplet for the mass conserving Stochastic Allen-Cahn equation, preprint.

5. Antonopoulou, D. C., Blomker, D. \& Karali, G. D., Front motion in the one-dimensional stochastic Cahn-Hilliard equation, SIAM J. Math. Anal. 44 (2012), 3242-3280. Zbl1270.35040 MR3023410

6. Antonopoulou, D. C. \& Karali, G. D., Existence of solution for a generalized Stochastic CahnHilliard Equation on convex domains, Discrete Contin. Dyn. Syst. B 16 (2011), 31-55. Zbl1227. 35163 MR2799541

7. Antonopoulou, D. C., Karali, G. D. \& Kossioris, G. T., Asymptotics for a generalized CahnHilliard equation with forcing terms, Discrete Contin. Dyn. Syst. A 30 (2011), 1037-1054. Zb11222. 35017 MR2812952

8. Caginalp, G., A conserved phase system: implication for kinetic undercooling, Phys. Rev. B 38 (1988), 789-891.

9. Caginalp, G., The dynamics of a conserved phase field system: Stefan like, Hele-Shaw, and CahnHilliard models as asymptotic limits, IMA J. Appl. Math. 44, (1990), 77-94. Zb10712. 35114 MR1044256

10. Caginalp, G. \& Chen, X., Convergence of the phase field model to its sharp interface limits, European J. Appl. Math. 9 (1998), 417-445. Zbl0930.35024 MR1643668

11. Cahn, J. W. \& Hilliard, J. E., Free energy of a nonuniform system, I. Interfacial free energy, J. Chem. Phys. 28 (1958), 258-267. 
12. Cahn, J. W. \& Hilliard, J. E., Free energy of a nonuniform system II, Thermodynamic basis, J. Chem. Phys. 30 (1959), 1121-1124.

13. CARdon-Weber, C., Cahn-Hilliard stochastic equation: Existence of the solution and of its density, Bernoulli 7 (2001), 777-816. Zb10995.60058 MR1867082

14. Carlen, E. A., Carvalho, M. C. \& Orlandi, E., Approximate solution of the Cahn-Hilliard Equation via corrections to the Mullins-Sekerka motion, Arch. Rat. Mech. Anal. 178 (2005), 1-55. Zb11076.76009 MR2188465

15. CHEN, X., Spectrum of the Allen Cahn, Cahn-Hilliard and phase field equations for generic interface, Comm. Partial Diff. Eqns. 19 (1994), 1371-1395. Zbl0811. 35098 MR1284813

16. CHEn, X., Global asymptotic limit of solutions of the Cahn-Hilliard equation, J. Differential Geom. 44 (1996), 262-311. Zbl :0874.35045 MR1425577

17. Cook, H., Brownian motion in spinodal decomposition, Acta Metallurgica 18 (1970), 297-306.

18. Da Prato, G. \& Debussche, A., Stochastic Cahn-Hilliard equation, Nonlin. Anal. Th. Meth. Appl. 26 (1996), 241-263. Zb10838.60056 MR1359472

19. Elliott, C. M. \& Zheng, S., On the Cahn-Hilliard equation, Arch. Rat. Mech. Anal. 96 (1986), 339357. Zb10624.35048 MR0855754

20. Escher, J. \& NishiURA, Y., Smooth unique solutions for a modified Mullins-Sekerka model arising in diblock copolymer, Hokkaido Mathematical Journal 31 (2002), 137-149 Zb11017. 35088 MR1888274

21. Escher, J. \& Simonett, G., Classical solutions of multidimensional Hele-Shaw models, SIAM J. Math. Anal. 28 (1997), 1028-1047. Zb10888. 35142 MR1466667

22. Evans, L. C., Partial differential equations, American Mathematical Society, 1998. Zbl0902. 35002 MR1625845

23. FunAKI, T., The scaling limit for a Stochastic PDE and the separation of phases, Probab. Theory Relat. Fields. 102 (1995), 221-288. Zb10834.60066 MR1337253

24. Gilbarg, D. \& Trudinger, N. S., Elliptic partial differential equations of second order, SpringerVerlag (1977) Zbl0361.35003 MR0473443

25. Gurtin, M. E., Generalized Ginzburg-Landau and Cahn-Hilliard equations based on a microforce balance, Physica D 92 (1996), 178-192. Zb10885. 35121 MR1387065

26. Hohenberg, P. C. \& Halperin, B. I., Theory of dynamic critical phenomena, J. Rev. Mod. Phys., 49 (1977), 435-479.

27. Katsoulakis, M. A. \& Vlachos, D. G., From microscopic interactions to macroscopic laws of cluster evolution, Phys. Rev. Letters 84 (2000), 1511-1514.

28. Kitahara, K., Oono, Y. \& Jasnow, D., Phase separation dynamics and external force field, Mod. Phys. Letters B 2 (1988), 765-771.

29. Novick-Cohen, A., The Cahn-Hilliard equation, Handbook of differential equations: evolutionary equations, Vol. 4, (2008) 201—228, Elsevier/North-Holland. Zbl1185. 35001 MR2508166

30. Omelyanov, G. A., Danilov, V. G. \& Radkevich, E. V. , Asymptotic solution of the conserved phase field system in the fast relaxation case, European Journal of Applied Mathematics 9 (1998), 1-21. Zbl0923.35082 MR1617005

31. Omelyanov, G. A., Danilov, V. G. \& Radkevich, E. V., Soliton type asymptotic solutions of the conserved phase field system, Portugaliae Mathematica 53 (1996), 471-501. Zb10880.35120 MR1432150

32. Omelyanov, G. A., Danilov, V. G. \& Radkevich, E. V., Tanh-type asymptotic solution of the conserved phase field system, Adv. Math. Sci. Appl 8 (1998), 663-689. Zb10921.35192 MR1657231

33. Pego, R. L., Front migration in the non-linear Cahn-Hilliard equation, Proc. R. Soc. Lond. A 422 (1989), 261-278. Zb10701.35159 MR0997638

34. Sтотн, B., Convergence of the Cahn-Hilliard equation to the Mullins-Sekerka problem in spherical 
symmetry, J Dif Eq. 125 (1996), 154-183. Zb10851. 35011 MR1376064

35. WANG, Q.-F. \& NAKAGIRI, S., Weak solutions of Cahn-Hilliard equations having forcing terms and optimal control problems, Mathematical models in functional equations (Kyoto, 1999), Sūrikaisekikenkyūusho Kōkyūroku, 1128 (2000), 172-180. Zbl0958. 35504 MR1777605 